\title{
Expert consensus and operational guidelines on exercise rehabilitation of chronic obstructive pulmonary disease with integrating traditional Chinese medicine and Western medicine
}

\author{
Xuan Chen ${ }^{1,2 \#}$, Di Gong ${ }^{2 \#}$, Huai Huang ${ }^{2 \#}$, Kexin Wang ${ }^{2 \#}$, Wei Zhang ${ }^{1}$, Shanqun Li ${ }^{2}$; on behalf of the \\ China COPD Respiratory Rehabilitation Writing Expert Group with integrating Traditional Chinese \\ and Western medicine, the Respiratory Disease Rehabilitation Professional Committee of China Medical \\ Education Association \\ ${ }^{1}$ Department of Respiratory and Critical Care Medicine, Shuguang Hospital, Shanghai University of Traditional Chinese Medicine, Shanghai, China; \\ ${ }^{2}$ Department of Pulmonary Medicine, Zhongshan Hospital, Fudan University, Shanghai, China \\ \#These authors contributed equally to this work. \\ Correspondence to: Dr. Shanqun Li. 180 Fenglin Road, Xuhui District, Shanghai 200032, China. Email: li.shanqun@zs-hospital.sh.cn; Dr. Wei Zhang. \\ 185 Pu'an Road, Huangpu District, Shanghai 200120, China. Email: zhangw1190@sina.com.
}

Submitted Mar 12, 2021. Accepted for publication May 21, 2021.

doi: 10.21037/jtd-21-431

View this article at: https://dx.doi.org/10.21037/jtd-21-431

\section{Introduction}

Chronic obstructive pulmonary disease (COPD) has become a widespread global chronic respiratory disease. In view of the large patient population, high mortality and disability rate, and heavy medical burden, COPD has become a major challenge for public health worldwide. According to epidemiological studies and demographic data, the prevalence of COPD in China is $13.7 \%$ and the number of COPD patients has reached more than 100 million. Therefore, it is of considerable significance to make more efforts to prevent and treat COPD (1).

COPD is a chronic respiratory disease with dyspnea as its main clinical manifestation, especially during physical activity. COPD is often associated with cardiovascular, motor, metabolic and other systemic diseases, and may lead to pathological changes in many other systems (2). Muscle dysfunction is the most common extrapulmonary change that may influence the skeleton and respiratory muscles. Muscle dysfunction can lead to amyotrophy, muscle weakness, and other symptoms, which influence the skeletal muscle most significantly and result in poor exercise tolerance in patients (3). The pathological changes in skeletal muscle and dyspnea reduce the participation of activities in COPD patients and cause secondary muscle atrophy. These factors can result in declining motor ability, which is the critical predictor of mortality in COPD patients (4).

COPD can influence patients' health and quality of life. Delaying disease progression, alleviating symptoms, improving psychological status, and enhancing quality of life are the main goals of medical interventions for COPD patients. Pulmonary rehabilitation, as an indispensable part of effective treatments, is a multidisciplinary management program targeted at COPD patients to control disease progression, improve quality of life, and reduce the hospitalization rate (5). Exercise treatment, as the core of rehabilitation programs, has a higher degree of evidence in pulmonary rehabilitation that can improve dyspnea symptoms, increase motor abilities, and be helpful for improving psychological disorders and enhancing quality of life $(3,6,7)$.

To better guide exercise rehabilitation treatment for COPD patients, and to provide a systematic and operational plan for community doctors, pulmonary doctors and other health care providers on rehabilitation treatment, evaluation, treatment and follow-up, an expert group on respiratory rehabilitation and a professional committee on respiratory rehabilitation of the China Medical Education Association formed a common understanding by searching 
domestic and foreign studies, consulting with experts, and discussion. This consensus is based on existing research to develop a declarative document, focusing on the feasibility of our current clinical reality. In clinical practice, we still need to consider each patient's specific situation to design individualized exercise rehabilitation programs.

We present the following article in accordance with the RIGHT reporting checklist (available at https://dx.doi. org/10.21037/jtd-21-431).

\section{Definition}

COPD is a normal disease that can be prevented and treated. It is characterized by persistent and progressive airflow limitation, with an increased chronic inflammatory response of airways and lungs to harmful particles or gases. Pulmonary rehabilitation is a comprehensive individual intervention program based on a comprehensive evaluation of the patient and disease characteristics. It includes not only exercise training, but also education and behavioral changes to improve the physical and mental health of patients with chronic respiratory disease and promote longterm adherence to healthy behaviors (8).

Pulmonary rehabilitation is applicable to a wide range of people and suitable for patients with different severity and stages. Even elderly patients can benefit from pulmonary rehabilitation (9). The Global Initiative for Chronic Lung Disease (GOLD) also recommends pulmonary rehabilitation for patients with severe symptoms and a high risk of acute exacerbation (10). Foreign guidelines recommend that clinicians should prescribe pulmonary rehabilitation for COPD patients with FEV1 <50\% Pred (11). Exercise therapy is the cornerstone of pulmonary rehabilitation and plays an important role in pulmonary rehabilitation programs.

\section{The therapeutic exercise of pulmonary rehabilitation process}

Exercise rehabilitation is applicable to COPD patients at all stages, and early intervention is recommended. Although the optimal time for patients with acute COPD exacerbations to start rehabilitation is unclear, the American Thoracic Society (ATS) and European Respiratory Society (ERS) (ERS/ATS) guidelines recommend pulmonary rehabilitation within 3 weeks of hospital discharge (2).

A series of procedures are included in the implementation of exercise rehabilitation programs. Clinical evaluation and functional assessment are important prerequisites to carry out rehabilitation programs, and then individual exercise prescription, training, and guidance are recommended. Therapeutic effects must be continuously followed up and observed (12) (Figure 1).

In view of the considerable heterogeneity in the basic condition and mobility of COPD patients, it is necessary to consider many related factors, such as ventilation ability and the function of the cardiovascular and muscle systems to promptly adjust the plan according to the disease development. In addition, COPD is a chronic wasting disease. With the progress of COPD, it affects the work ability and economic level of patients. The guides focus on the assessment process of the basic condition of COPD patients, and introduce in detail various Chinese and Western exercise prescriptions that are beneficial to pulmonary rehabilitation, which have been used to guide health care providers and patients to choose suitable and affordable exercise rehabilitation programs.

\section{Patient assessment}

Limitation of activities in COPD patients is often influenced by many factors. Before the exercise rehabilitation intervention, patients should be evaluated from many aspects, including medical history, physical examination, dyspnea symptoms, lung function, exercise capacity, and nutritional status to elucidate the factors and provide a basis for formulating an exercise prescription. Throughout this process, some complications that may lead to exercise intolerance can be ruled out, such as cardiovascular disease, thus improving exercise safety.

\section{Medical history}

A detailed understanding of the COPD patient's medical history is essential for selecting the correct exercise rehabilitation programs. Therefore, it is necessary to pay more attention to the severity of symptoms, complications, laboratory examination data, and treatments history. Moreover, further information on drug treatment history, oxygen therapy, and other relevant details is helpful to ensure exercise safety. For example, in patients with diabetes, special attention should be paid to therapeutic regimens, especially insulin, to prevent exercise-induced hypoglycemia and the risk of ketosis in patients with type 1 diabetes. 


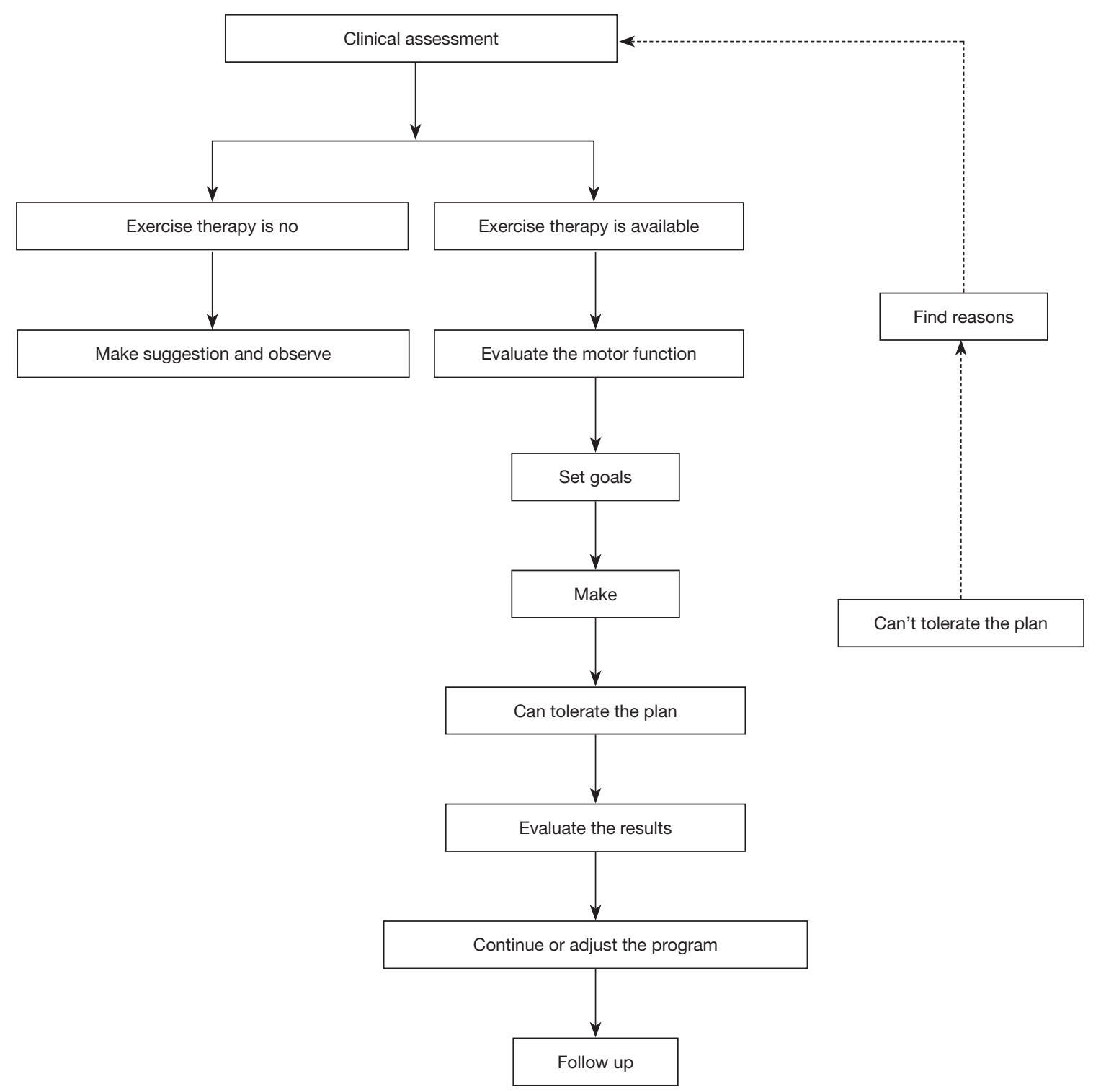

Figure 1 The procedures of exercise rehabilitation program.

\section{Physical examination}

A thorough and complete physical examination allows the doctor to determine if the patient is unfit for exercise rehabilitation. For COPD patients, examinations should focus on kinematic characteristics in the lungs, including the degree of emphysema, the mobility of the diaphragm, the manner of breathing, and the distribution, nature, and intensity of lung rales. Physical signs related to cardiopulmonary function including the heart size, the nature and loudness of heart sounds and murmurs, the liver size, hepatojugular reflux syndrome, and lower limb edema should be seriously considered.

\section{Dyspnea assessment}

Dyspnea is an important factor affecting the motor ability of COPD patients. Although breathing difficulty is associated with physiological abnormalities, it can also be regarded as a primarily subjective experience that can be influenced by many factors, such as anxiety, depression, sadness, fear, or previously acute dyspnea experience. 
Table 1 The Medical Research Council dyspnea scale

\begin{tabular}{ll}
\hline Grade & Degree of breathlessness related to activity \\
\hline 1 & Not troubled by breathless except on strenuous exercise \\
2 & Short of breath when hurrying on a level or when walking up a slight hill \\
3 & Walks slower than most people on the level, stops after a mile or so, or stops after 15 minutes walking at own pace \\
4 & Stops for breath after walking 100 yards, or after a few minutes on level ground \\
5 & Too breathless to leave the house, or breathless when dressing/undressing \\
\hline
\end{tabular}

The permission is granted from the MRC to use MRC Dyspnoea Scale for any purpose of research according to the MRC'S Open Access Policy. MRC, Medical Research Council.

Scoring tables are commonly used to evaluate the degree of dyspnea occurring in daily activities, such as the San Diego Dyspnea Questionnaire and the Medical Research Council (MRC) Dyspnea Scale. The MRC is relatively simple and widely used in clinical research. In this scale, the symptoms up to grade 2 are moderate and grades 3-5 are severe (Table 1). The dyspnea score during exercise rehabilitation is an important reference for formulating an exercise rehabilitation program with appropriate intensity. The Visual Analogical Dyspnea Score (VAS) and Borg Rating Scale are commonly used to monitor dyspnea during exercise testing and training. The Borg dyspnea scale is shown in Table S1.

\section{Respiratory function assessment}

Pulmonary function tests are necessary for respiratory disease and of considerable clinical value for detecting lung and airway diseases at an early stage, assessing the severity, prognosis, and therapeutic effect of disease, differentiating reasons for dyspnea, and locating the disease region. Pulmonary function tests include pulmonary volume and capacity, bronchial provocation and dilation, and diffusion function and airway resistance tests. Standard lung function index abnormalities are helpful for determining the diagnosis and physiological abnormalities. However, the disease severity in COPD patients is not limited to a single physiological lung function index, and it is not appropriate to predict a patient's response to exercise rehabilitation by just measuring the degree of airflow limitation (13). Additionally, evaluating respiratory muscle function is also an important part of assessing respiratory function. The maximal aspiration pressure (MEP) and maximal inspiration pressure (MIP) can reflect muscle strength, thus providing a reference for the proper muscle training load.

\section{Motor ability assessment}

\section{Muscle strength}

Muscle strength assessment is designed to measure the muscles' ability to exert force during exercise rehabilitation and is one of the tests that can reflect the functional state of muscle. Muscle strength is an important basis for recommending exercise rehabilitation programs. The manual muscle test (MMT) is a simple qualitative test and can be used as a fast bedside evaluation method (Table S2). Quantitative tests require some external equipment and the testing standards are high. For example, isokinetic muscle strength testing systems and grip dynamometers can be used to assess the contractility of the targeted muscle group. One repetition maximum (1-RM) is the maximum weight that a muscle can resist during a complete contraction. The 1-RM test can assess the muscle's ability to resist resistance during the action performed and needs the assistance of external devices. The 1-RM test result is an important strength training reference index.

\section{Exercise endurance}

Exercise endurance assessment is an important basis for establishing individualized aerobic exercise prescriptions that can be used to evaluate exercise safety and the influencing factors of limited exercise. The cardiopulmonary exercise test (CPET) and walk test are currently primarily used in clinical practice (Table 2) $(3,14)$.

The CPET is very valuable for exercise rehabilitation prescriptions. COPD patients often suffer exercise intolerance caused by single or multiple physiologic problems; using the CPET can detect hypoxemia, arrhythmias, musculoskeletal disorders, and cardiac ischemia during exercise rehabilitation. VO2max, WRmax, and other variables obtained via the symptom-limited 
Table 2 Endurance tests for COPD patients

\begin{tabular}{lll}
\hline Items & Observation indexes & Test equipment \\
\hline Incremental exercise tests & $\begin{array}{l}\text { VO2peak, VO2max, WRmax, lactate threshold, } \\
\text { fatigue score, pulse oxygen saturation }\end{array}$ & $\begin{array}{l}\text { Cycle ergometer or treadmill, pneumatometer, heart } \\
\text { monitor, pulse oximeter }\end{array}$ \\
Fixed-power exercise test & Time, fatigue score, pulse oxygen saturation & $\begin{array}{l}\text { Cycle ergometer or treadmill, pneumatometer, heart } \\
\text { monitor, pulse oximeter }\end{array}$ \\
Shuttle walking test & $\begin{array}{l}\text { Time, distance, pulse oxygen saturation, degree of } \\
\text { dyspnea }\end{array}$ & 10-meter trail and pulse oximeter \\
Endurance shuttle walking test & $\begin{array}{l}\text { Time, distance, pulse oxygen saturation, degree of } \\
\text { dyspnea }\end{array}$ & 10-meter trail and pulse oximeter \\
6-minute walking distance & $\begin{array}{l}\text { Distance, pulse oxygen saturation, degree of } \\
\text { dyspnea }\end{array}$ & 30-meter trail and pulse oximeter \\
\hline
\end{tabular}

VO2peak, peak oxygen uptake; VO2max, maximum oxygen uptake; WRmax, maximum rate of work.

CPET are important exercise intensity parameters. The CEPT including incremental load or constant load tests, cycle ergometers, and treadmill tests are common methods. The former is relatively simple, requires less space, and is more common in clinical practice (14). Another exercise test commonly used is the walking test, which is also very simple to perform and does not require additional equipment. This test can be conducted under non-laboratory conditions and demonstrates exercise rehabilitation outcomes with more sensitivity (3). The 6-minute walking and shuttle walking tests are more commonly used in clinical research.

\section{Nutritional status assessment}

Nutritional status is an indicator to judge the prognosis and guide exercise rehabilitation. Malnutrition is also an independent risk factor for poor prognosis (15). In COPD patients, weight loss is common, and causes include unbalanced energy metabolism, inflammatory muscle changes, and other factors. In addition, the body composition also changes, especially a decrease in lean body weight, while those conditions may be improved by exercise rehabilitation therapy to a certain extent. Commonly used nutritional assessment indicators are body mass index (BMI) and body composition. BMI is calculated as weight $(\mathrm{kg}) /$ height $(\mathrm{m})^{2}$. BMI $<21 \mathrm{~kg} / \mathrm{m}^{2}$ is low weight, $21 \leq \mathrm{BMI}$ $\leq 25 \mathrm{~kg} / \mathrm{m}^{2}$ is normal weight, and BMI $>30 \mathrm{~kg} / \mathrm{m}^{2}$ is overweight. Bioelectrical impedance is used to evaluate the patient's fat-free mass (FFM).

\section{Components of exercise rehabilitation prescription}

Patients with different types of COPD have considerable dysfunction heterogeneity. No exercise rehabilitation prescription is currently recommended according to different stages or treatment plans, and individual exercise rehabilitation prescriptions are also formulated according to the principles of exercise rehabilitation and prescription guidelines, including frequency, intensity, time, and type, also known as FITT. The duration of exercise rehabilitation training has also not been unified. It is believed that a longer exercise rehabilitation training period will provide more lasting clinical improvements. Therefore, to achieve better physical outcomes, it is recommended that COPD patients should work on their exercise rehabilitation training at least 3 times a week for 6 to 12 weeks under professional supervision $(5,16)$.

\section{Exercise rehabilitation intensity}

For the exercise rehabilitation intensity of COPD patients, disease severity, degree of respiratory limitation, complications, and enthusiasm for sports are important to consider, and the intensity should be promptly adjusted according to changes in their condition. Generally, to improve aerobic exercise capacity and muscle strength, exercise rehabilitation intensity should be higher than daily exercise. If the initial intensity can be tolerated, patients can gradually increase exercise rehabilitation intensity 
Table 3 The exercise rehabilitation intensity rating scale

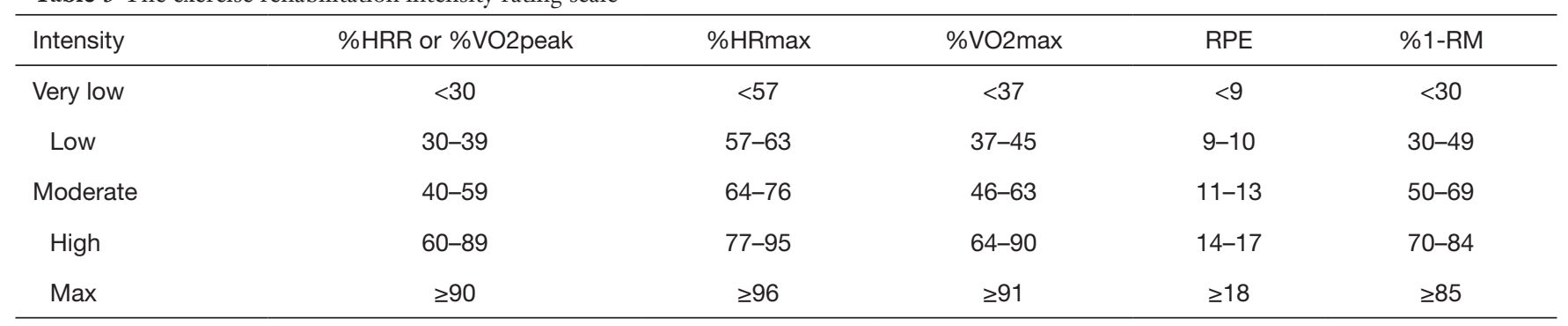

HRR, heart rate reserve; VO2peak, peak oxygen uptake; VO2max, maximum oxygen uptake; RPE, rating perceived of exertion; 1-RM, one repetition maximum.

Table 4 Different types of exercise rehabilitation therapy

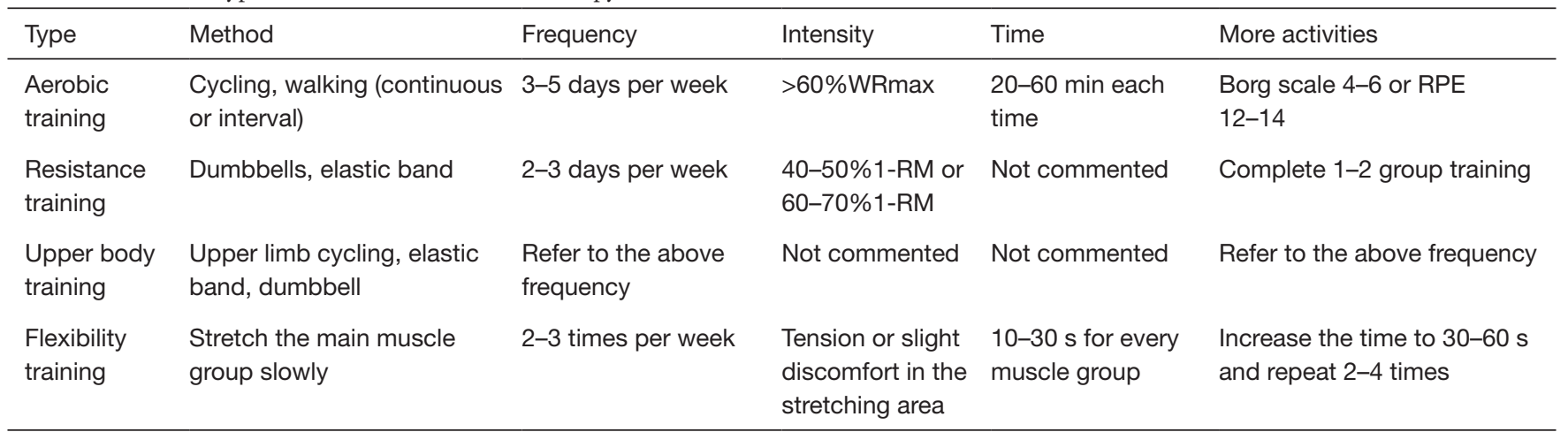

with no obvious discomfort. Although high-intensity training is beneficial for patients to produce more positive physiological changes, some symptoms of respiratory constraints may appear before the expected physiological changes occur. Therefore, for most patients, low and moderate intensity training is more significant to achieve long-term compliance. The exercise rehabilitation intensity setting should refer to the exercise capacity evaluation, such as VO2max, HR, 1-RM, and metabolic equivalent. In addition, exercise rehabilitation intensity can also be evaluated and monitored according to fatigue scores, such as rating perceived exertion (RPE) and symptoms during and after exercise. The exercise rehabilitation intensity grading standards recommended by the American College of Sports Medicine can serve as a reference for clinicians to develop an appropriate exercise regimen for COPD patients (Table 3) $(17,18)$.

\section{Types of exercise rehabilitation therapy}

Types of exercise rehabilitation therapy vary. Aerobic, resistance, and breathing training are commonly used in clinical practice. Other training methods such as nerve and muscle electrical stimulation, whole body vibration training, and balance training have recently been reported, but in clinical practice, former methods and flexibility training remain common and are often combined with multiple exercises. Applying traditional Chinese exercise rehabilitation therapy for COPD rehabilitation also has its own characteristics and is attracting increasing attention. Exercise programs for the development of cardiopulmonary fitness, muscle strength and flexibility have been recommended by the American College of Sports Medicine, the Thoracic Society and the European Thoracic Society, etc., which can be used as a reference for the formulation of exercise prescriptions for patients with COPD patients (Table 4) (19).

\section{Factors to consider during exercise rehabilitation}

Exercise rehabilitation prescription should be based on the patient's motor capacity. In addition to the general situation, basic activity, and degree of ADL limitation, patients' preferences, financial status and availability of 
exercise equipment should also be considered, which can encourage patients to exercise. Moreover, patients can train under the guidance of medical monitoring and personnel if conditions permit. Patients with stable conditions but who cannot train regularly in hospitals due to factors such as traffic can be guided remotely to train properly at home and in the community.

Safety should be monitored during exercise rehabilitation interventions, especially in patients with unstable cardiovascular function or active bleeding and other exercise contraindications. Once the above situation occurs, it is necessary to receive active clinical treatment first, and then resume exercise rehabilitation intervention after the condition is stable (20). Hypoxemia is an important exercise intolerance factor in COPD patients. For patients with hypoxemia, blood oxygen saturation should be monitored during exercise and oxygen administration is suggested to maintain $\mathrm{SpO}_{2}$ above $90 \%$ and reduce discomfort caused by exercise $(19,21)$

\section{Common methods of exercise rehabilitation}

\section{Aerobic training}

Aerobic training includes long-term rhythmic exercises in which the body's main muscle groups participate under adequate oxygen supply conditions. Aerobic training improves patients' endurance and heart and lung function. There are many kinds of aerobic training, such as walking, jogging, cycling, aerobics, and traditional Chinese exercises such as Tai Chi. Aerobic training consists of both continuous and interval training. Interval training reaches the maximum intensity through a series of short, highintensity training periods regularly interspersed with lowintensity training and rest. For example, patients can start at $80-100 \%$ WRpeak for 20 seconds. They then rest for 40 seconds and repeat for three to four cycles to make the total exercise time roughly equivalent to continuous exercise. The power can be increased by an additional $5-10 \%$ when patients can tolerate that intensity. Interval exercise can achieve the same exercise effect as continuous exercise and the subjective discomfort caused by interval exercise is tolerable $(5,22)$. Clinicians often recommend interval exercise if patients' FEV $1<40 \%$ Pred,

WRpeak $<60 \%, \mathrm{SpO}_{2}<85 \%$, steady-state power test for less than 10 minutes, and suffer difficulty breathing during exercise, which can be used as a reference in clinical research (21).
Exercise training can be conducted outdoors or indoors with fixed-power bicycles, treadmills, and other equipment. In upper limb power bicycle training, an arm dynamometer is fixed at the level of the patient's shoulder, and patients turn a crank with both arms to perform a bicycle-like movement. In lower-limb power bike training, the seat height should be adjusted and maintained at the thigh level. Patients should keep their shoulders back, stomach in, jaw slightly closed, and eyes facing forward, body in a neutral position, and hip joints stable during training. They should start with 5-10 minutes of low-intensity exercise, then 20-30 minutes of designated-intensity exercise, and finally 5-10 minutes of low-resistance and slow-speed exercise to cool down. In this training, the exercise rehabilitation intensity can be regulated by changing the speed or resistance (23).

Stair climbing is also a form of aerobic training that can be conducted with stairways or simulated stairs. Climbing stairs is also a daily functional activity that is more specific for patients who demand more daily activities. Before training, a complete evaluation of lower limb joint function is necessary, especially the knee joints. This training may increase the likelihood of joint and muscle pain, so it should be avoided if patients have severe lower joint disorders. In addition, when stair climbing is a training method, it is also necessary to determine the exercise rehabilitation intensity based on cardiopulmonary exercise rehabilitation assessment and monitor fatigue and the degree of dyspnea during training to control the intensity.

\section{Resistance training}

The decline of skeletal muscle strength is an important cause of decreased activity and exercise tolerance in COPD patients. Resistance training is an active exercise performed by muscles when they resist gravity or overcome external resistance. It can restore and develop muscle strength and has positive effects on improving muscle endurance. Resistance exercise consumes less oxygen, requires less ventilation per minute, and has relatively low respiratory pressure, so it is a better choice for patients with endstage lung disease or other complications. The upper and lower limb muscle groups can both be trained by strength training. In international reports, the recommended level of lower limb strength training for respiratory rehabilitation is Grade 1A (24). There are many ways to achieve strength training, such as grip dynamometers, elastic bands, and weight resistance training (dumbbells and barbells). Body weight can also be used in training, such as squatting. The 
exercise load can be increased along with increasing exercise tolerance. It is also recommended that resistance training combined with regular aerobic training can further improve muscle strength (8). The strength training program is described in Table 4.

\section{Flexibility training}

Flexibility training can increase and maintain the body's flexibility and joint motion through gentle muscle stretching and slow-motion exercises, which facilitate the completion of daily activities and reduce injuries. Flexibility training is also one of the practical exercises of cardiopulmonary diseases and can be combined with other exercise training, but there is no further research on COPD patients (19). The clinical flexibility training program is described in Table 4.

\section{Upper body training}

Upper body training is necessary for COPD patients as upper limb activities are particularly important for independent daily life. For example, dressing, bathing, and daily household chores require upper limb participation. The diaphragm's mechanical efficiency decreases in patients due to lung hyperinflation. Therefore, accessory inspiratory muscles such as the latissimus dorsi, trapezius, and pectoralis major and minor muscles assist ventilation to some extent. When these muscle groups are used for daily upper limb movements, the diaphragmatic load will increase and has a higher demand for metabolism and ventilation, thus resulting in irregular, shallow, and uncoordinated breathing and dyspnea. These symptoms can be effectively improved by training and the upper body movement ability in COPD patients can also be enhanced (25). However, it is unknown whether upper body training is more effective for improving health-related quality of life (26).

Upper body training also includes aerobic and resistance training. At present, there is no recommendation for the best program for COPD patients. According to whether the upper limb is supported, upper body training is also divided into unsupported and supported training. Unsupported upper body training focuses on resistance training of the biceps, deltoids, triceps, and trapezius muscles using dumbbells. Generally, the initial load is $50 \% 1-\mathrm{RM}$, and the intensity can be gradually increased if the Borg score $\leq 3$ (25). Upper body exercises can be supporting using a cycle ergometer or rowing machine. Studies have shown that six weeks of upper body aerobic training in patients with COPD can increase endurance, regulate hyperventilation, and significantly reduce minute ventilation under the same conditions (27).

\section{Breathing training}

Breathing training is also an important component of exercise rehabilitation for COPD patients. It can be combined with other exercise rehabilitation training to establish an effective breathing mode, improve breathing and respiratory muscle function, reduce symptoms, and improve exercise tolerance (16).

\section{Pursed lip breathing}

Pursed lip breathing increases airway pressure by tightening the lips, prevents the small airway from collapsing and closing, and reduces functional residual capacity. Breathing patterns are simultaneously improved, muscles are relaxed, and breathing times are prolonged, which increases the tidal volume, improving ventilation function and the gas exchange process (28). Patients with moderate to severe COPD are most likely to benefit from this type of breathing training $(29,30)$.

Training method: (I) ask the patient to assume a comfortable position, maintaining the upper body perpendicular to the ground (standing or sitting). (II) Relax the neck and shoulder muscles. (III) Close the lips and inhale through nose for at least 2 seconds. (IV) Contract the lips as a "whistle" to force air through the mouth slowly for at least 4 seconds (time it by silently counting). (V) Keep the stomach in and force more air out of the lungs. The proper ratio of inspiration time to expiration time is $1: 2$, the rate of respiration is $8-10$ times/min, and the exercise should continue for 15-20 minutes each session.

\section{Abdominal breathing}

Abdominal breathing training is also known as phrenic breathing training. In COPD patients, the diaphragm is compressed by over-expanded lungs and flattened. The diaphragm's activity and the efficiency of its contractions also decrease. In severe cases, the diaphragm is so weak that contradictory movements of the chest and abdomen appear. The key to abdominal breathing is to coordinate the activities of the diaphragm and abdominal muscles during breathing.

Patients should assume a comfortable position, relax their whole body, close their mouth, and breathe deeply through their nose until they cannot breathe more, then hold their breath for a few seconds if possible and slowly exhale through their mouth. When people inhale, the diaphragm 
descends and the abdomen protrudes, while the opposite occurs during exhalation. The patient should place both hands under the rib bow to feel it sinking and expanding as they inhale and exhale. If necessary, press the lower ribs and abdomen with both hands to promote abdominal muscle contractions and exhalation.

Abdominal breathing training can be done in a seated or standing position and combined with walking training. Patients can inhale and exhale according to a certain rhythm, such as two-step breathing, four-step breathing, or breathing in and out at a ratio of 3:2 during walking exercise. The most important factor is ensuring that the symptoms of dyspnea do not deteriorate during long walking periods.

\section{Abdominal breathing with load \\ Adding loads to combat abdominal distension during abdominal breathing can improve the diaphragm's function. For example, patients can lie in a supine position with flexed knees and hip joints, and a sandbag is placed on their abdomen. The sand bag's weight is determined by the weight that allows 10 complete abdominal breathing repetitions, also called 10-RM, meaning the maximum diaphragm contraction for 10 repetitions. To increase muscle strength, the training weight used should be set at $60 \%, 75 \%$, and $100 \%$ of $10-\mathrm{RM}$ for 10 repetitions in each group for a total of three groups, while 10-15 minutes at $35-75 \%$ of $10-\mathrm{RM}$ is required in endurance training.}

\section{Resistance training of inspiratory muscles}

Inspiratory muscle weakness often occurs in COPD patients, especially in moderate and severe patients. Researchers found that inspiratory muscle resistance training can improve symptoms and motor function (31). Inspiratory muscle training can be added to systemic training programs. To achieve the desired results, this training requires resistance or threshold pressure loading devices. The initial training intensity should be set to at least $30 \%$ PImax, and the resistance load should be gradually increased as the tolerance increases. Resistance training of the inspiratory muscles is recommended for 15 min twice a day (32).

\section{Representative exercise rehabilitation therapy for pulmonary rehabilitation in traditional Chinese medicine (TCM)}

TCM exercise rehabilitation therapies include flexible body postures, coordinated stretching of the body muscles with one's own weight, and coordination of breathing methods.
Patients can obtain good pulmonary rehabilitation effects without resorting to medical rehabilitation equipment and paying additional costs. In addition, it can be practiced alone or in groups, which is conducive to improving the mental health of patients $(33,34)$.

\section{Tai Cbi}

Tai Chi is a series of slow and rhythmic circular movements that change from one form to another. Studies have shown that Tai Chi can reach $50-60 \%$ of heart rate reserves in those 25-80years of age (35). Therefore, Tai Chi is considered a low-intensity aerobic exercise. Studies have shown that Tai Chi can improve 6-minute walking distances and $\mathrm{FEV}_{1} \%$ compared to other regular exercise, but its effect on improving breathing and quality of life has yet to be confirmed (36). The Tai Chi practice time depends on the complexity and number of movements. The simplified version of Tai Chi released by the General Administration of Sport of China consists of 24 movements, including parting the wild horse's mane; white crane spreads its wings, brush knee, and side steps, among others. Actions such as hold, stroke, push, press, and others are mainly used in Tai Chi to attack and defend. In addition, the Tai Chi movements are soft, emphasizing consciousness to guide breathing and cooperating with the entire body. Detailed Tai Chi movements are shown in Figures 2-17.

\section{Ba Duan Fin}

Abdominal breathing is used in Ba Duan Jin. It requires breathing through the abdomen and maintaining deep, long, thin, slow, average, and soft breaths. With the cooperation of diaphragm and thorax movement, this exercise can promote inhaling more fresh air, exhaling residual gas, increasing the effective cavity, promoting alveolar expansion, and eventually improving pulmonary function in COPD patients (37). Furthermore, the chest muscles can be strengthened through many upper limb movements. Ba Duan Jin can help patients breathe, form a natural state of relaxation with the body and spirit, cultivate the mind, stabilize the emotions, and balance and relieve anxiety. It can eliminate negative emotions and improve quality of life. Detailed Ba Duan Jin movements are shown in Figures 18-22.

\section{Liu Zi fue}

This training for good health maintenance is an ancient 


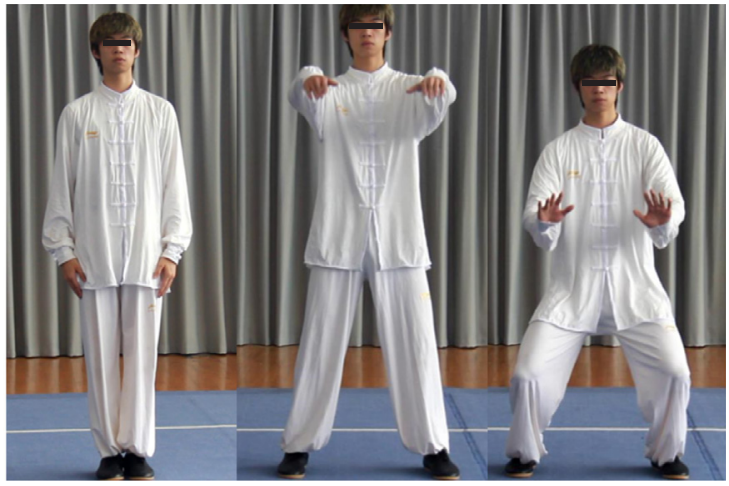

Figure 2 The diagram of simplified Tai Chi (1 style). The detailed actions of Tai Chi: Beginning.

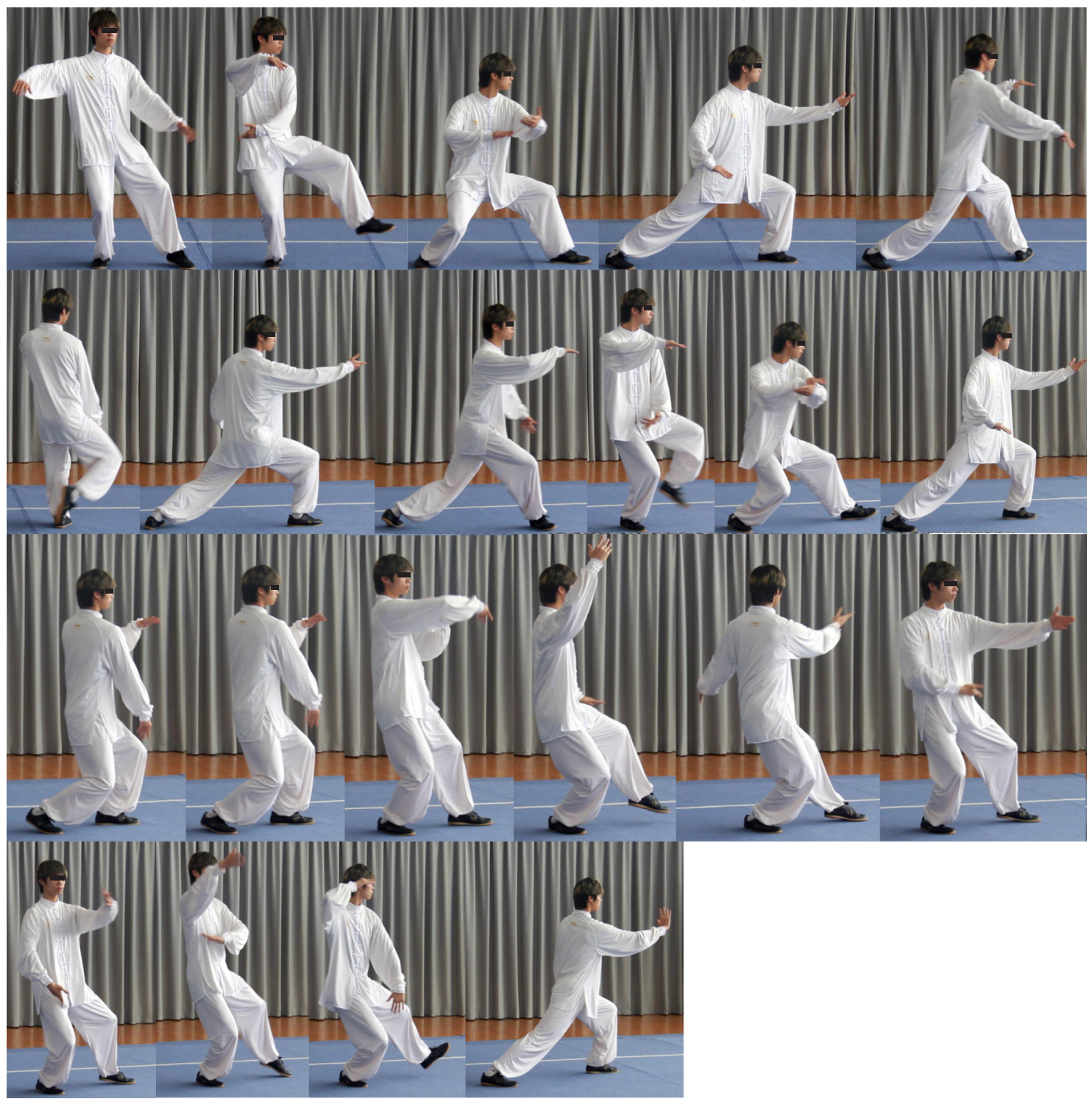

Figure 3 The diagram of simplified Tai Chi (2 style). The detailed actions of Tai Chi: Parting the Wild Horse's Mane (3 times). 

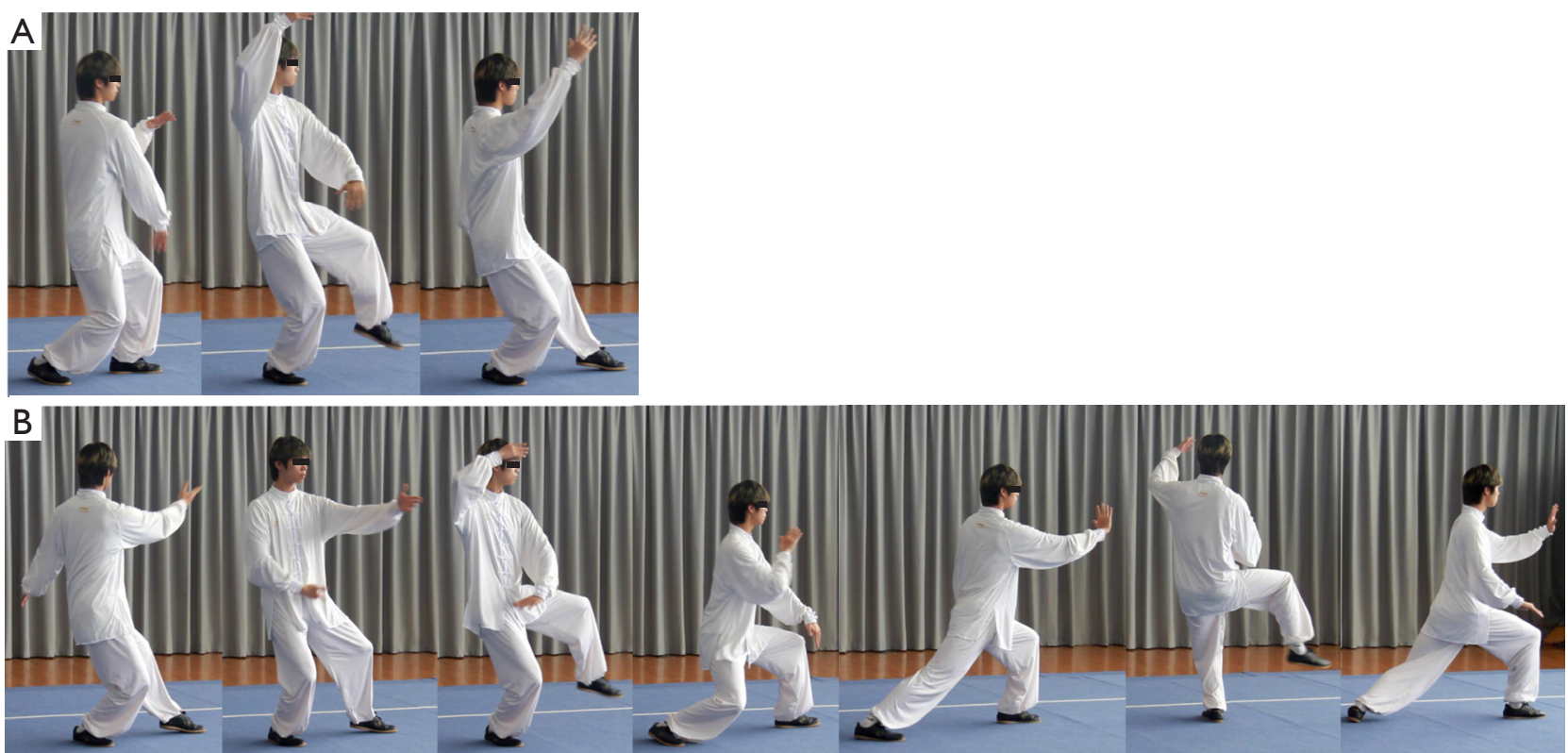

Figure 4 The diagram of simplified Tai Chi (3 to 4 styles). The detailed actions of Tai Chi: (A) White Crane Spreads its Wings. (B) Brush Knee and Side Step (3 times).
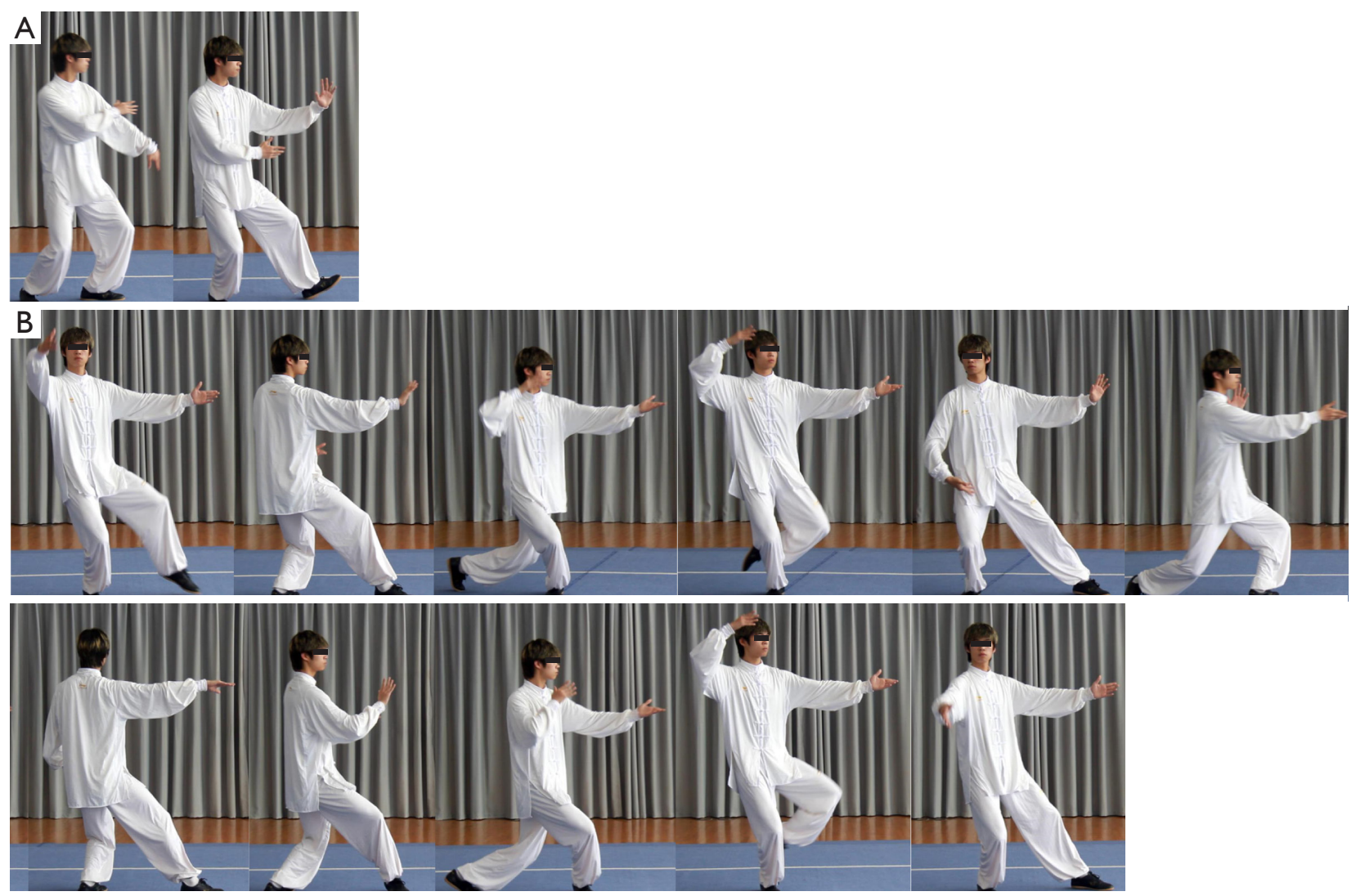

Figure 5 The diagram of simplified Tai Chi (5 to 6 style). The detailed actions of Tai Chi: (A) Play the Lute. (B) Step Back and Repulse Monkey (4 times). 


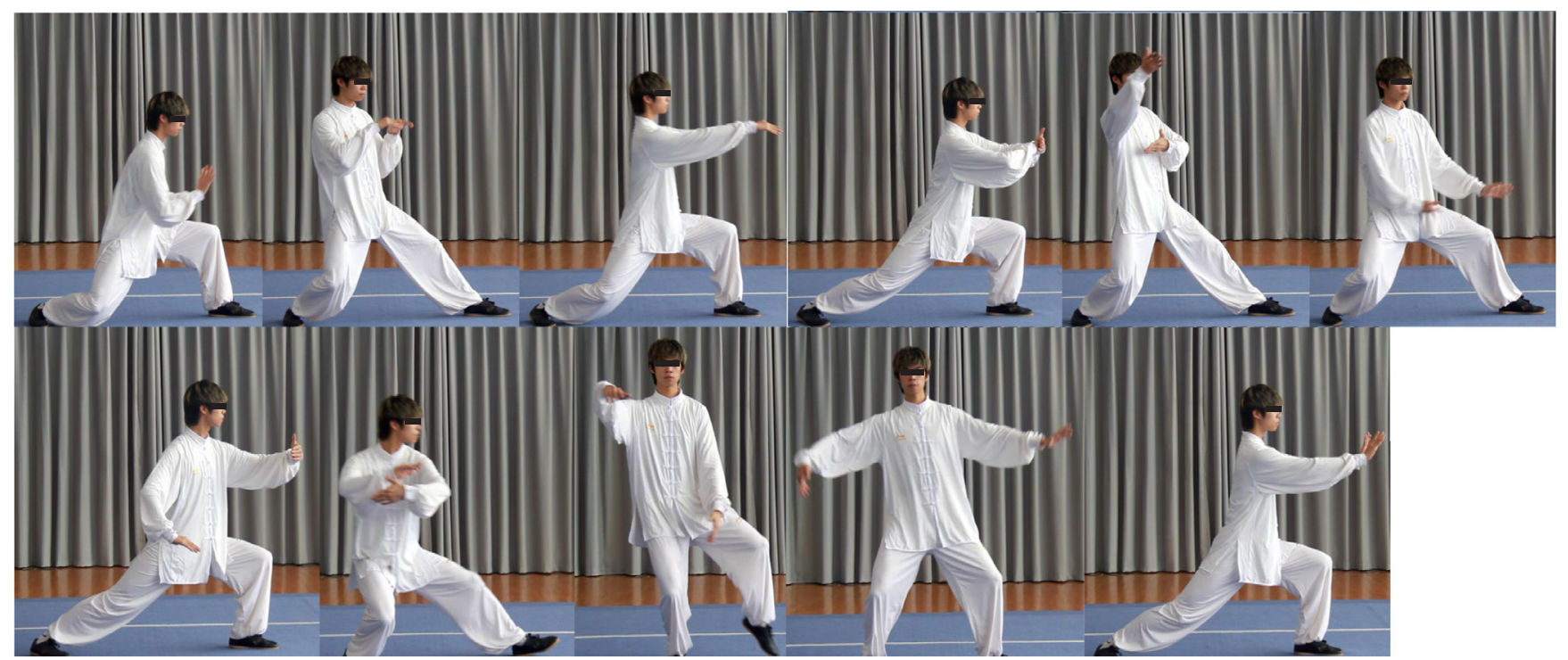

Figure 6 The diagram of simplified Tai Chi (7 style). The detailed actions of Tai Chi: Grasp the Sparrow's Tail-left.

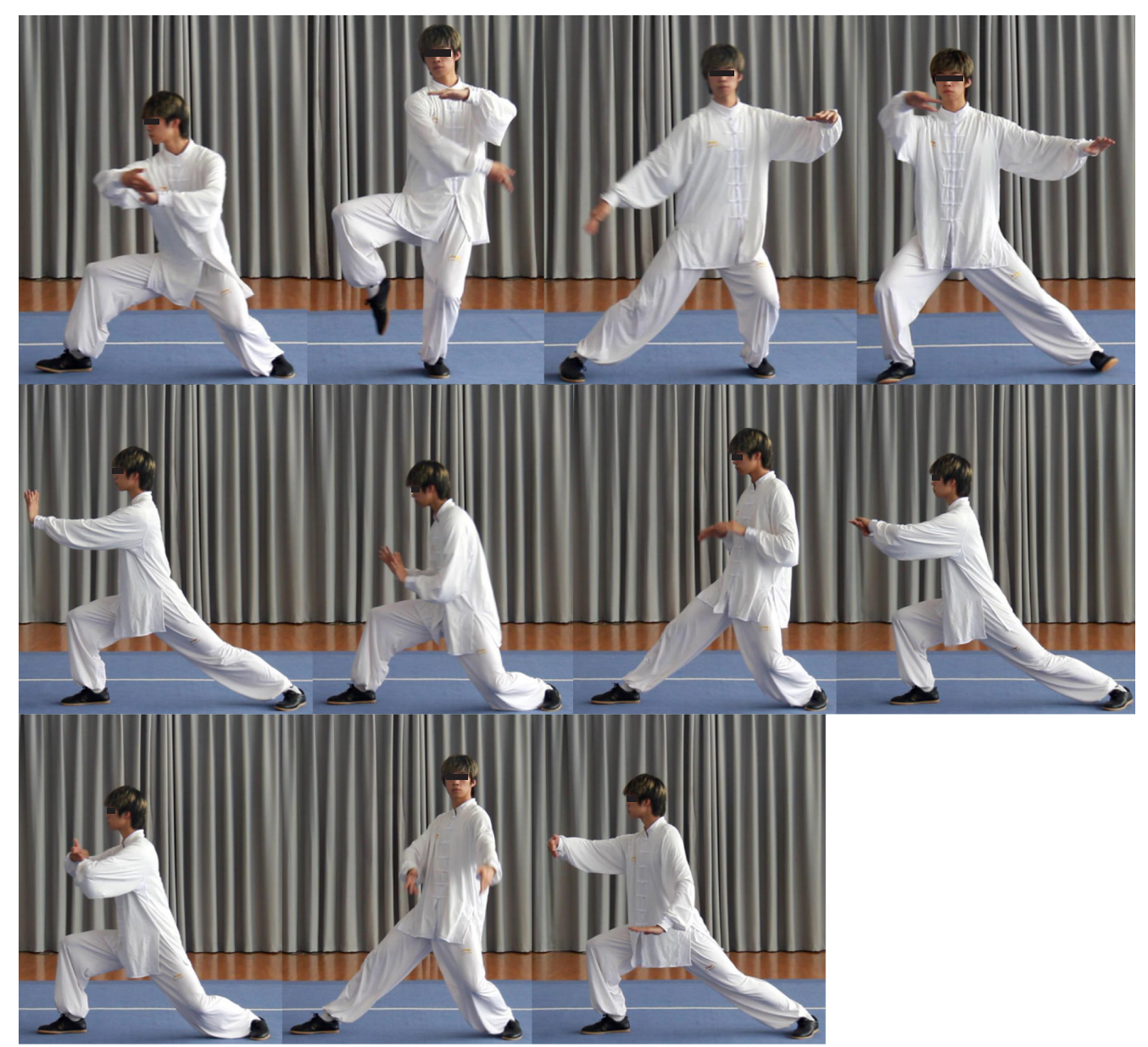

Figure 7 The diagram of simplified Tai Chi (8 style). The detailed actions of Tai Chi: Grasp the Sparrow's Tail—right. 


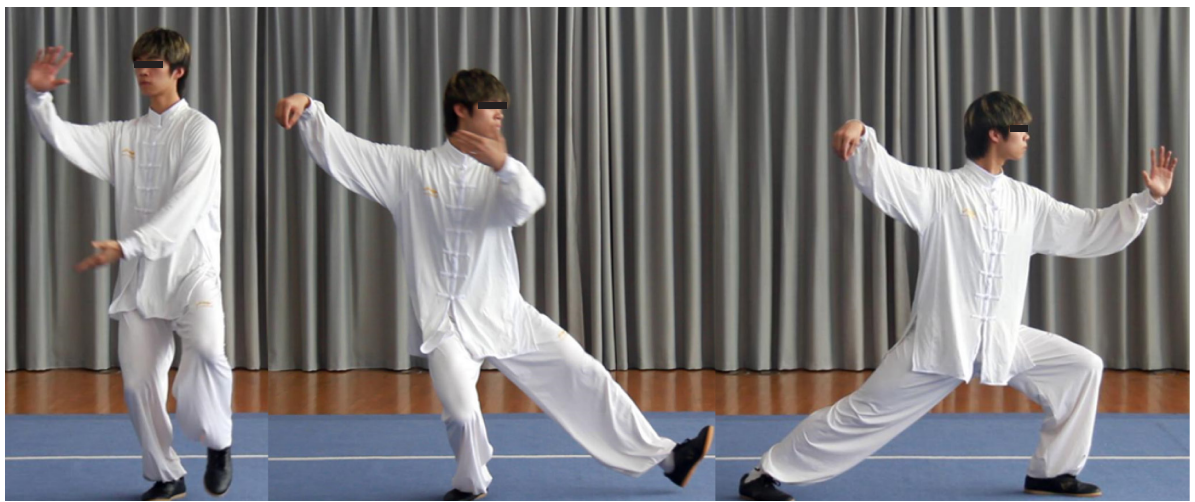

Figure 8 The diagram of simplified Tai Chi (9 style). The detailed actions of Tai Chi: Single Whip.

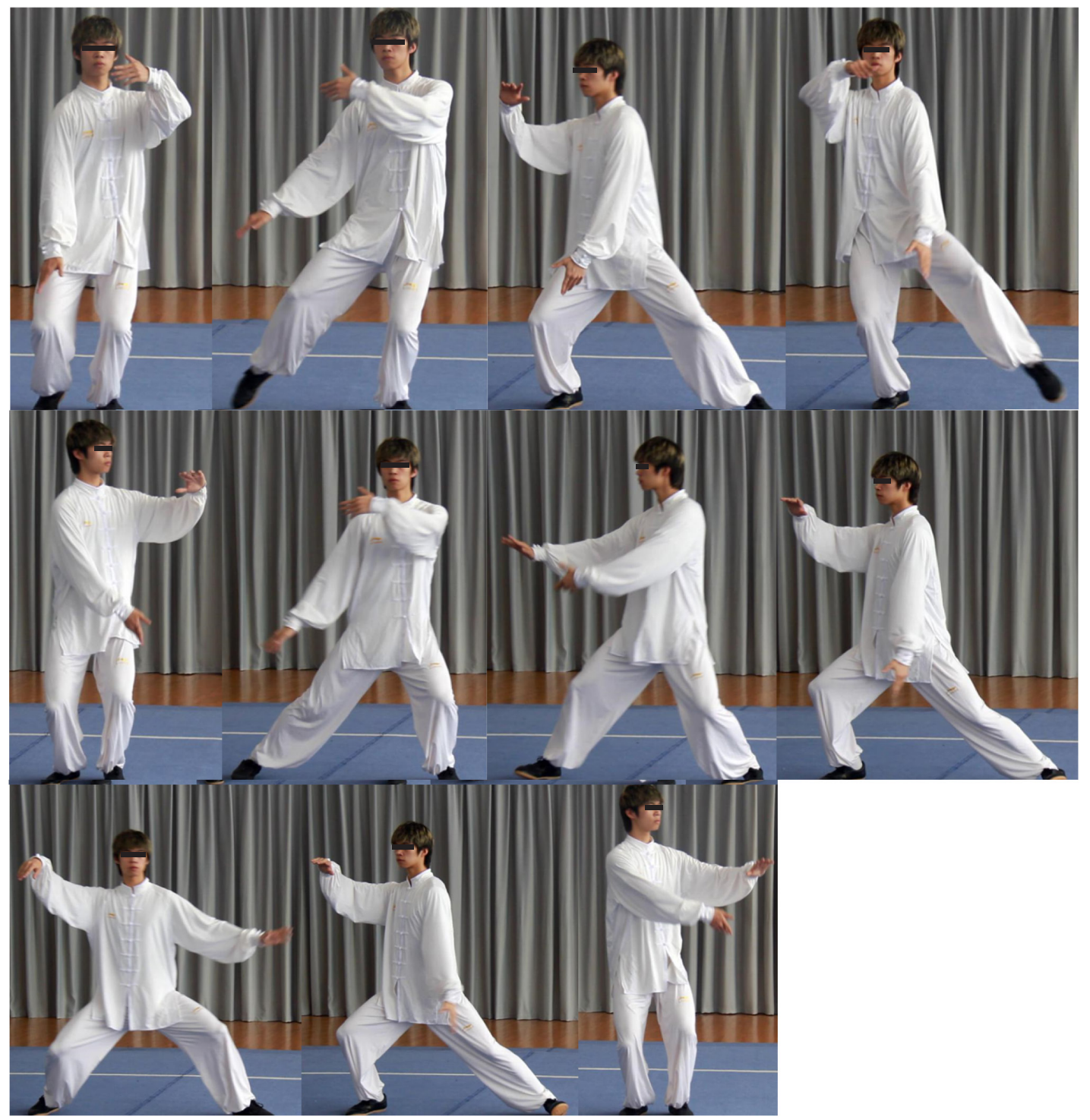

Figure 9 The diagram of simplified Tai Chi (10 style). The detailed actions of Tai Chi: Wave Hands Like Clouds (3 times). 

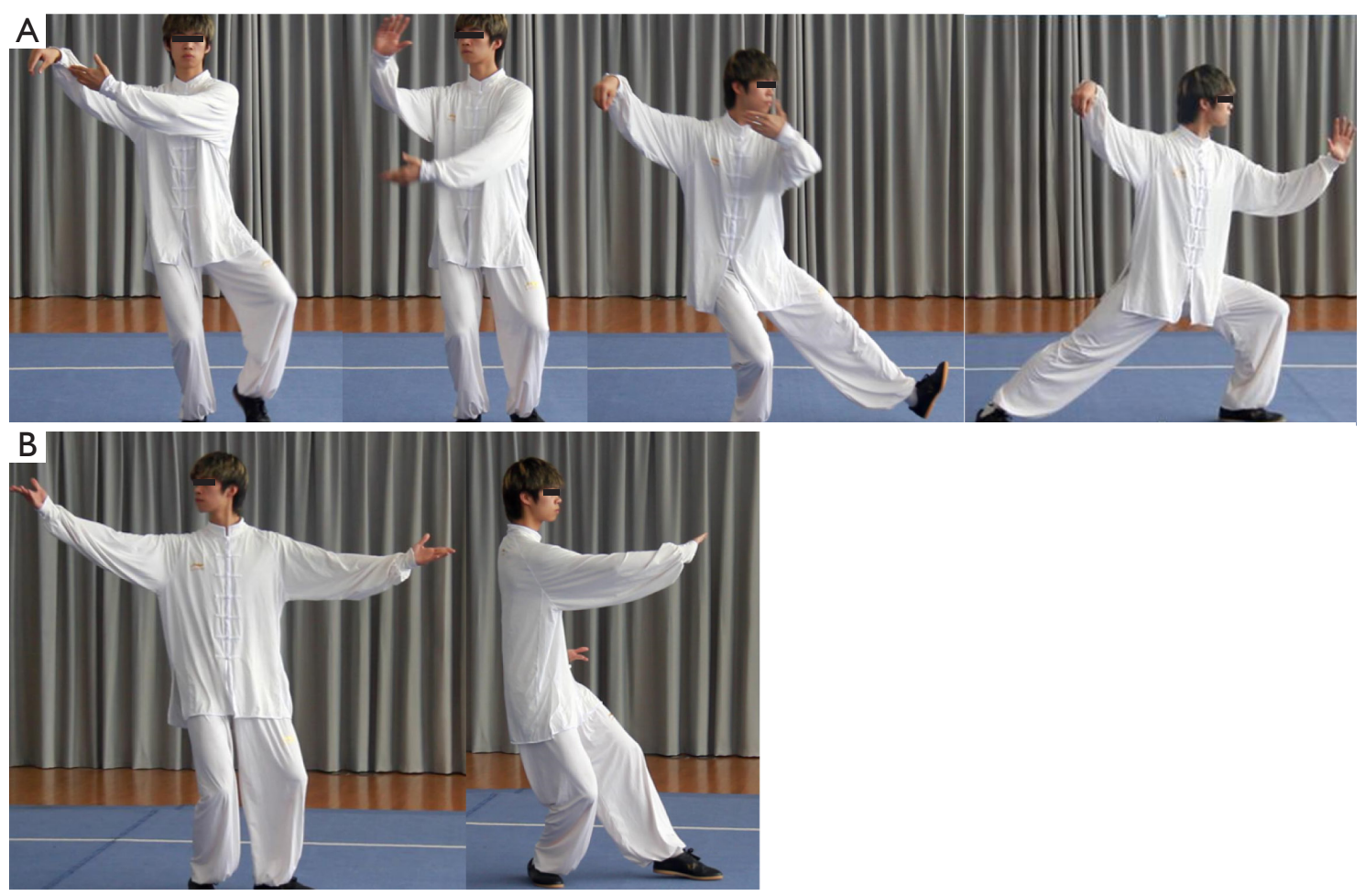

Figure 10 The diagram of simplified Tai Chi (11 to 12 styles). The detailed actions of Tai Chi: (A) Single Whip. (B) High Pat on Horse.
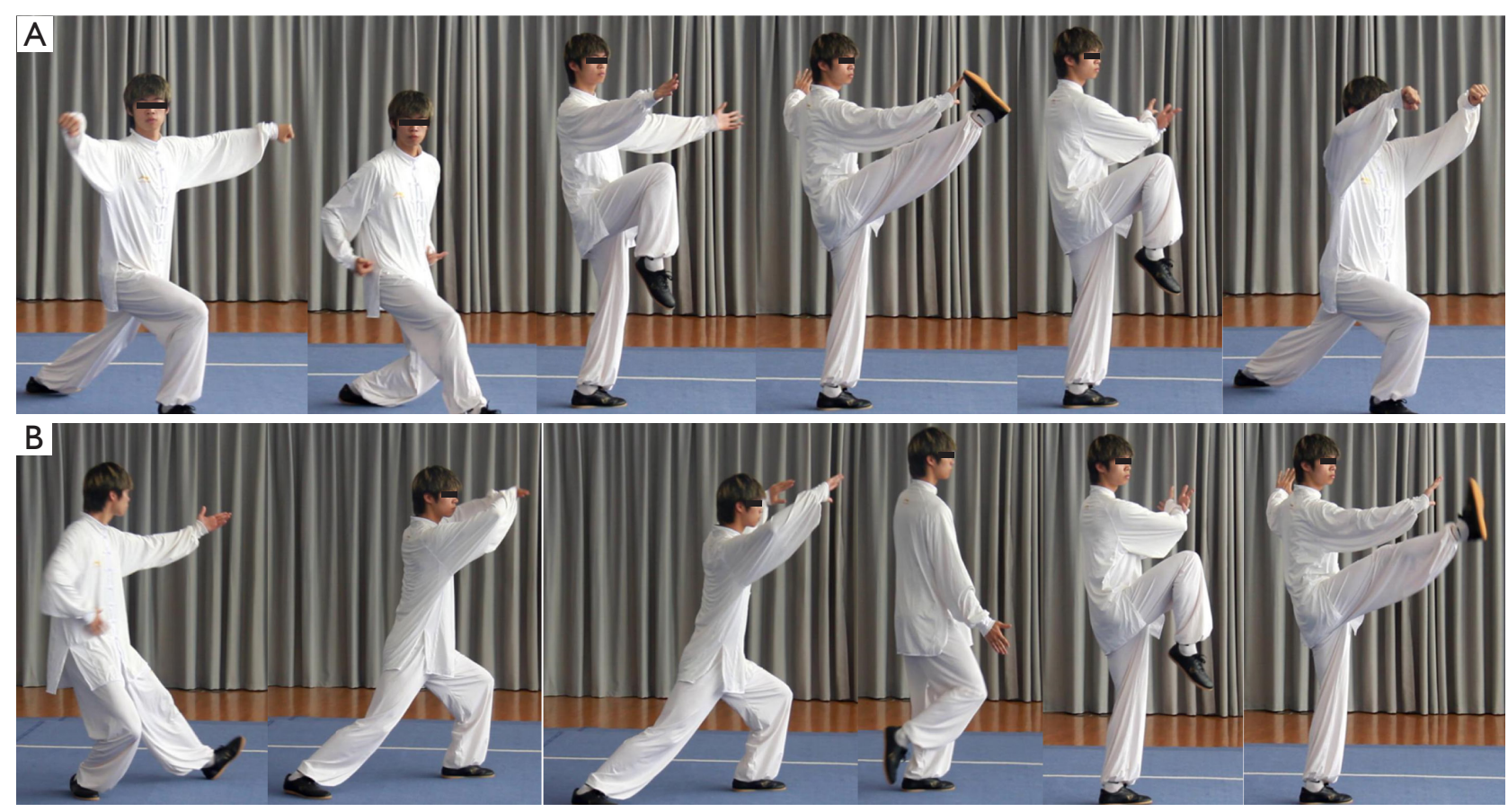

Figure 11 The diagram of simplified Tai Chi (13 to 14 styles). The detailed actions of Tai Chi: (A) Kick With Right Heel. (B) Strike Ears With Fists. 

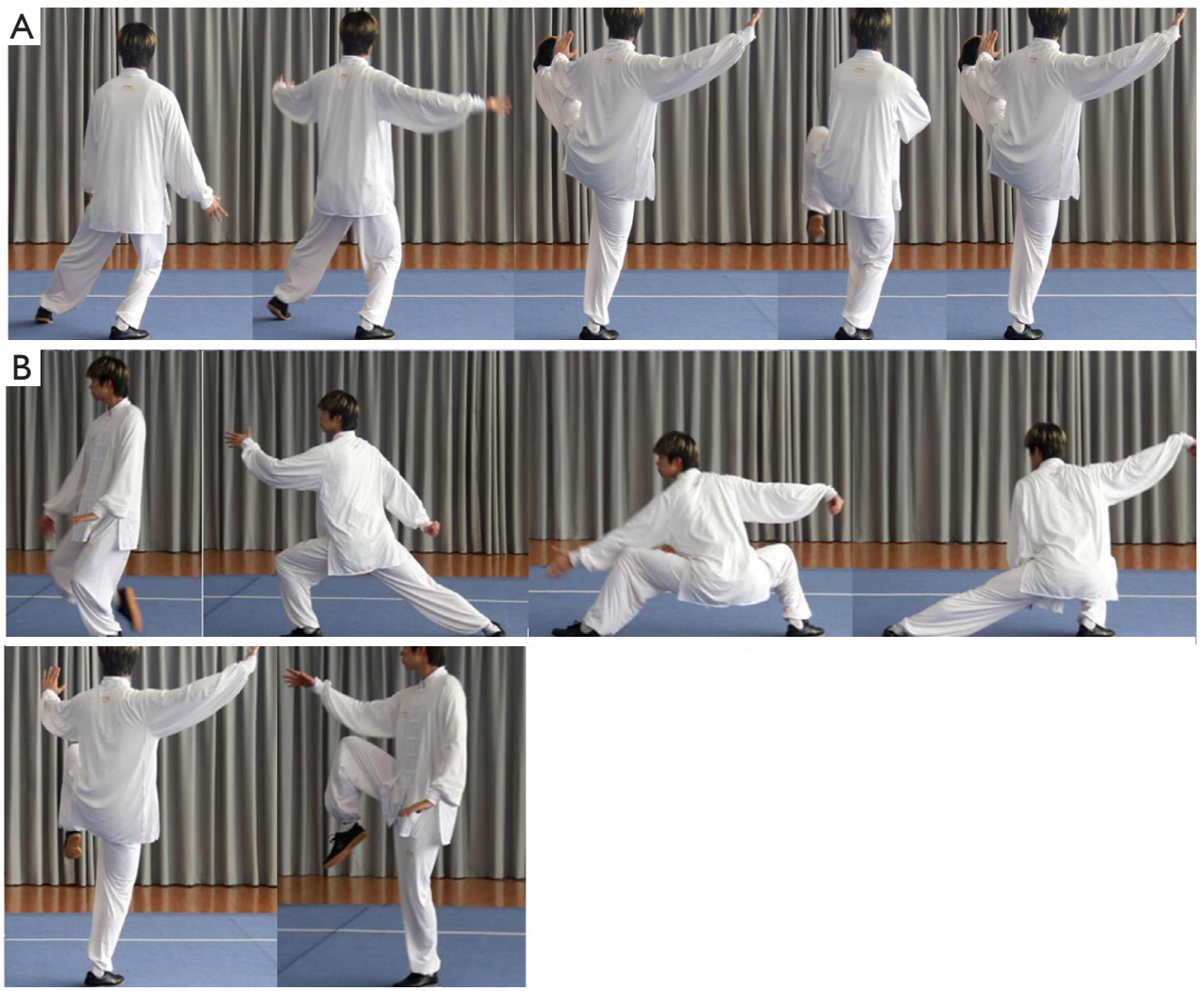

Figure 12 The diagram of simplified Tai Chi (15 to 16 styles). The detailed actions of Tai Chi: (A) Kick With Left Heel. (B) Snake Creeps Down (left).
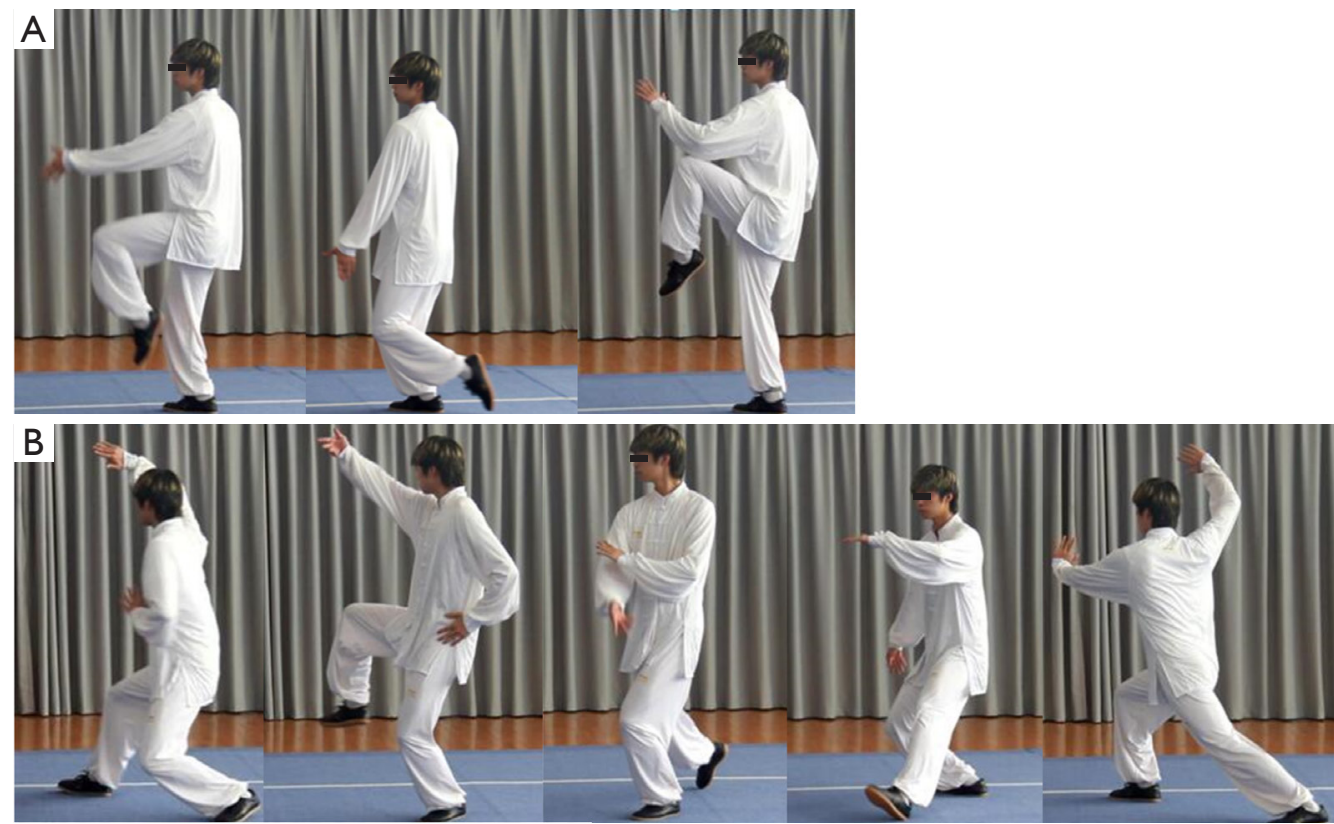

Figure 13 The diagram of simplified Tai Chi (17 to 18 styles). The detailed actions of Tai Chi: (A) Snake Creeps Down (right). (B) Fair Lady Weaves the Shuttle (right and left). 

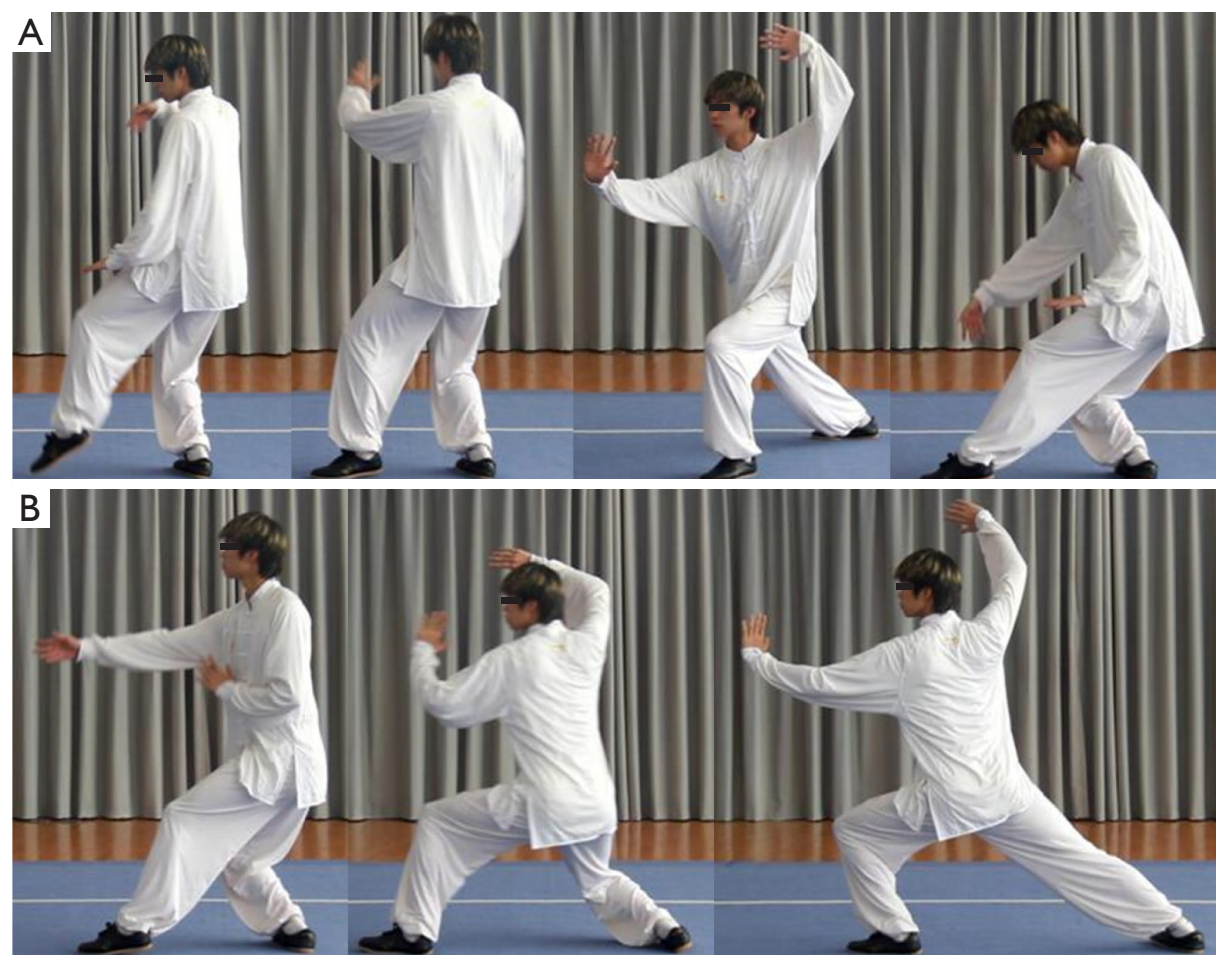

Figure 14 The diagram of simplified Tai Chi (19 to 20 styles). The detailed actions of Tai Chi: (A)Needle at the Bottom of the Sea. (B) Fan through Back.

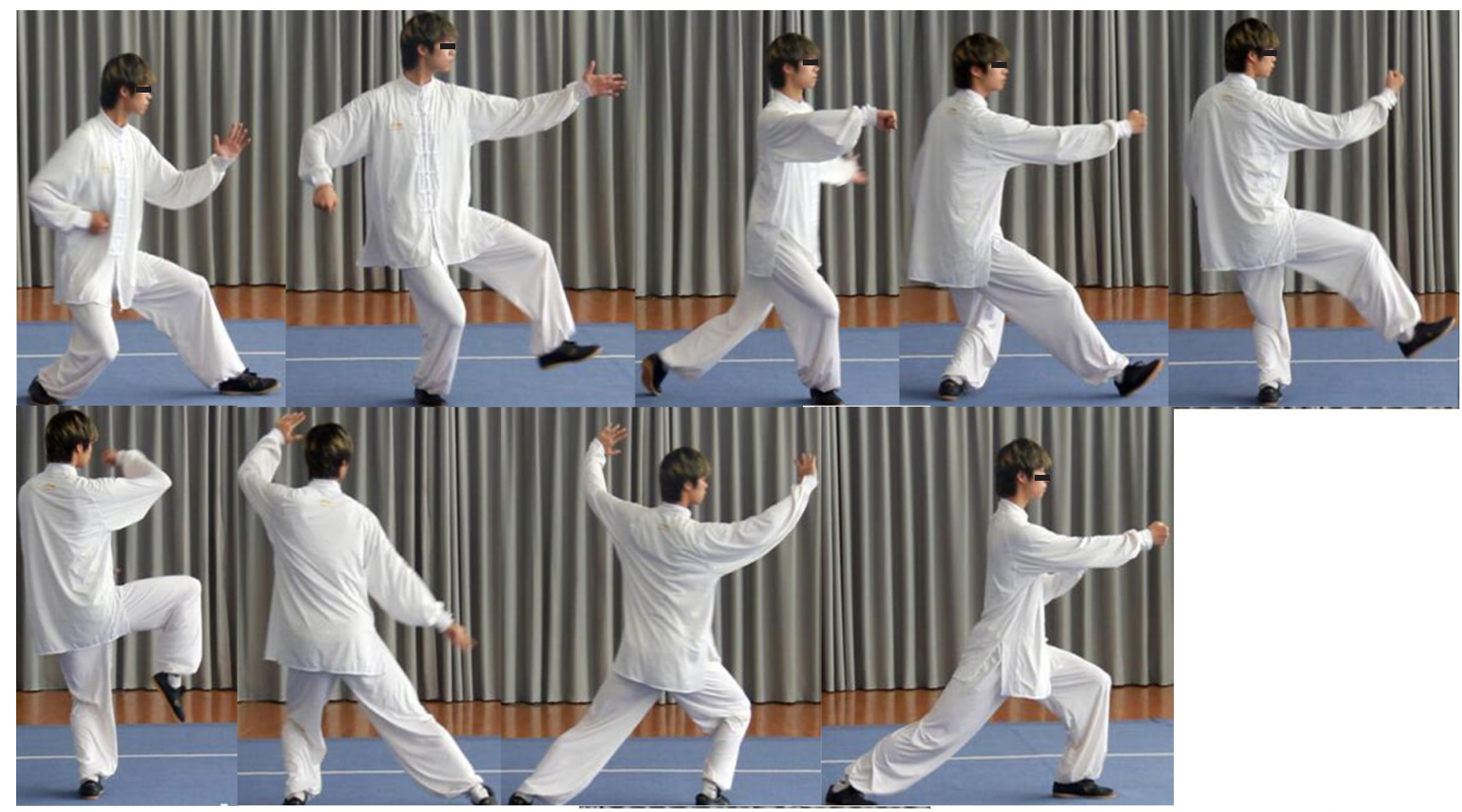

Figure 15 The diagram of simplified Tai Chi (21 style). The detailed actions of Tai Chi: Deflect Downwards, Parry and Punch. 

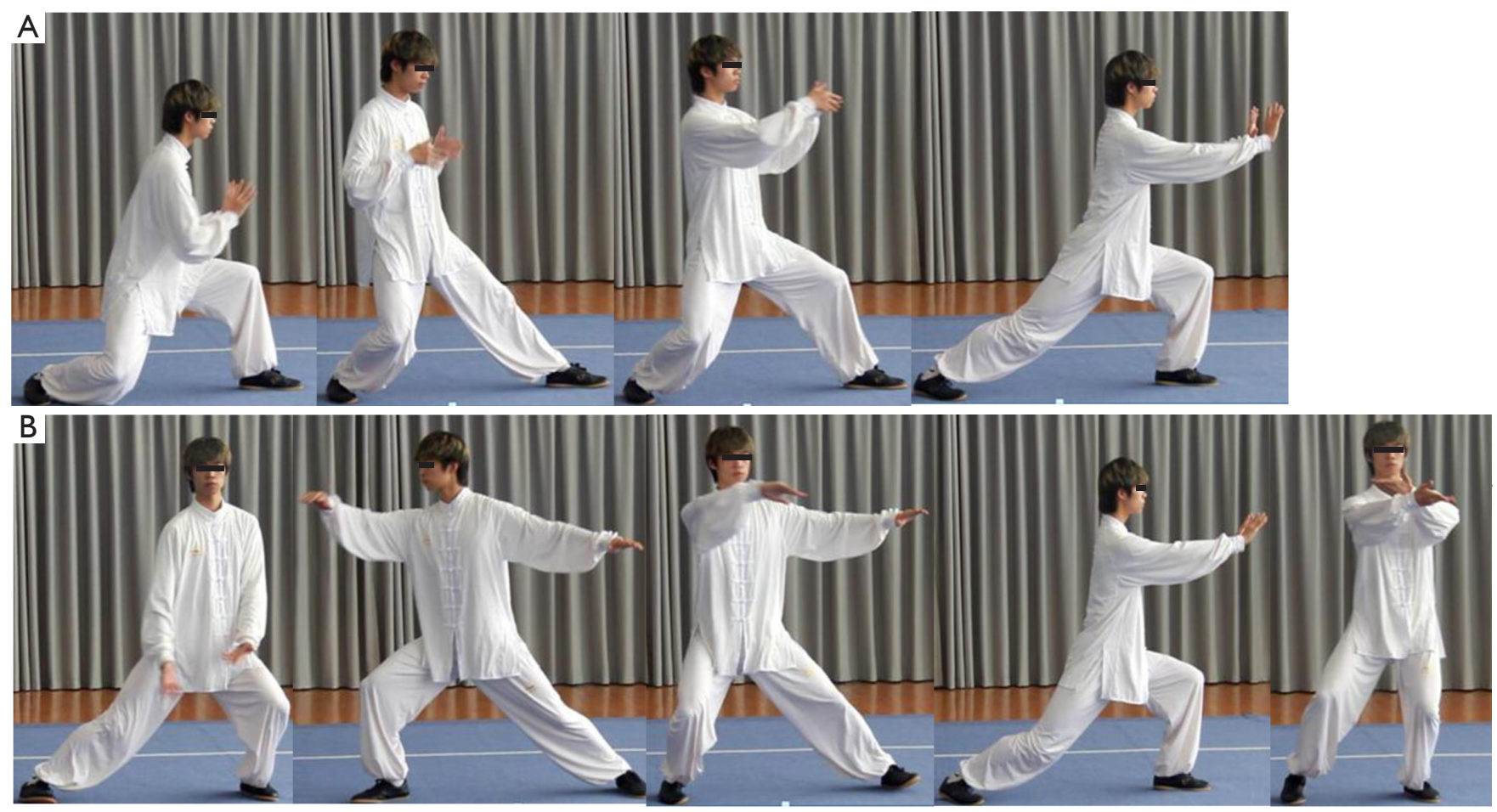

Figure 16 The diagram of simplified Tai Chi (22 to 23 styles). The detailed actions of Tai Chi: (A) Apparent Close Up. (B) Cross Hands.

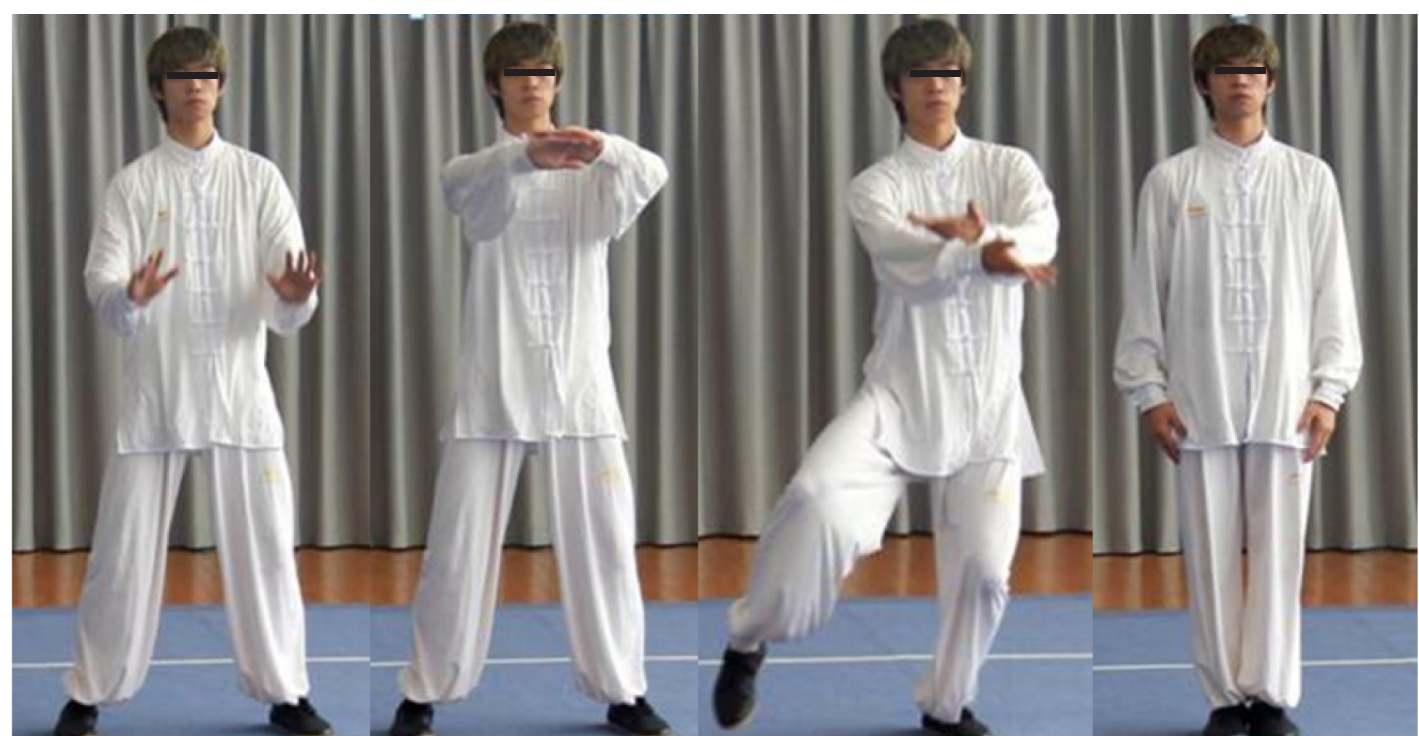

Figure 17 The diagram of simplified Tai Chi (24 style). The detailed actions of Tai Chi: Closing Form.

Chinese method. The breathing method helps regulate the systemic circulation and functions of different organs to some extent using six healing sounds of six characters: $\mathrm{Xu}$, $\mathrm{He}, \mathrm{Hu}, \mathrm{Xi}$, Chui, and Xi. The six characters correspond to the meridians of the lung, heart, spleen, liver, kidney, and triple energizer, respectively. Liu $\mathrm{Zi}$ Jue is the healthy Qigong that matches the body movement and breathing. During this type of training, the rate and depth of breathing 

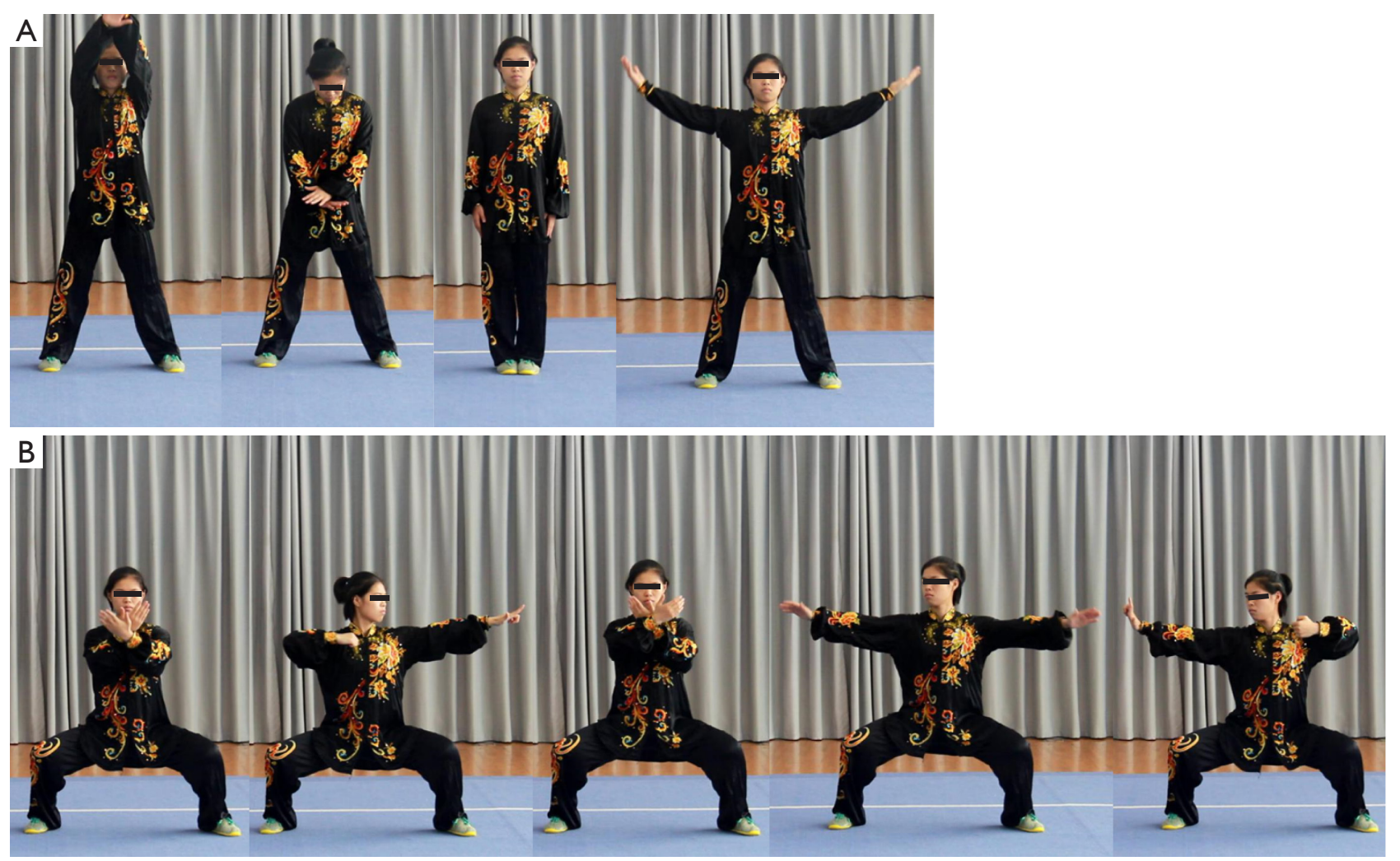

Figure 18 Ba Duan Jin (1-2 styles). The detailed actions of Ba Duan Jin: (A) Push up the sky to regulate "tri-jiao". (B) Draw a bow on both side like shooting a vulutre.

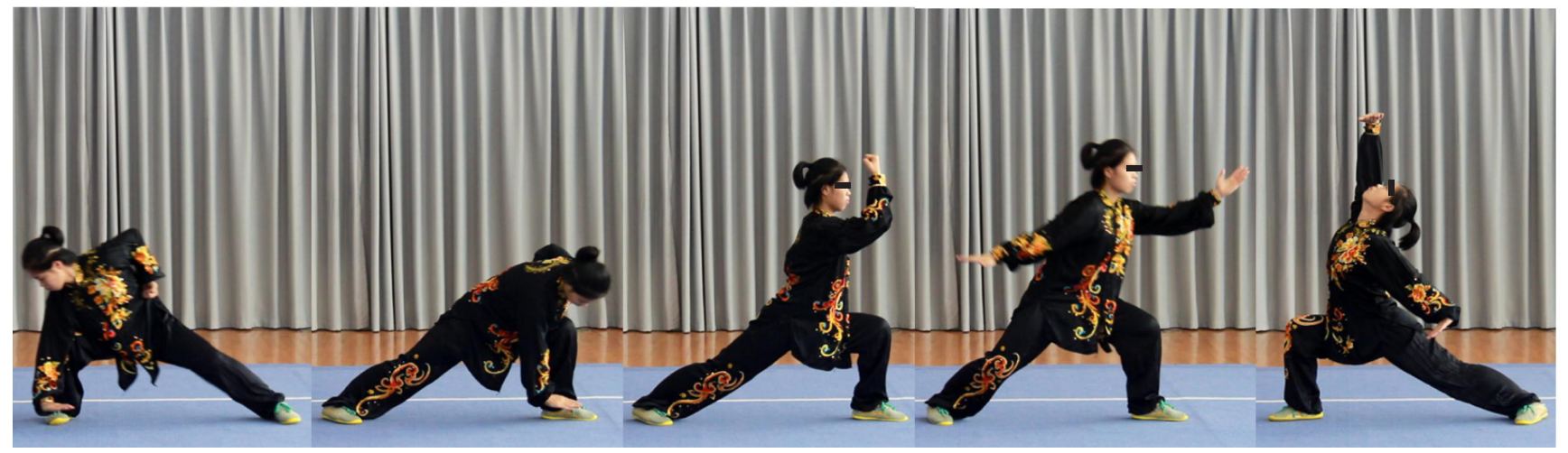

Figure 19 Ba Duan Jin (3 style). The detailed actions of Ba Duan Jin: Raise single arm up to regulate spleen (Pi) and stomach (Wei).

increase, similar to the ventilation. Deep abdominal breathing can increase the diaphragm's range of motion, strengthening the diaphragm and auxiliary muscles as well as increasing alveolar wall elasticity. Liu $\mathrm{Zi}$ Jue can improve the six-minute walking distance and quality of life in stable COPD patients (38). In 2003, the General Administration of Sports revised it and promoted it nationwide.

The Liu $\mathrm{Zi}$ Jue training method is as follows: It is performed in a certain order, such as commencing from $\mathrm{Xu}-\mathrm{He}-\mathrm{Hu}-\mathrm{Xi}-\mathrm{Chui}-\mathrm{Xi}$ and closing. Liu $\mathrm{Zi}$ Jue includes 9 movements as follows: $\mathrm{Xu}$ sound training in the spring improves vision, He sound training in the summer protects 


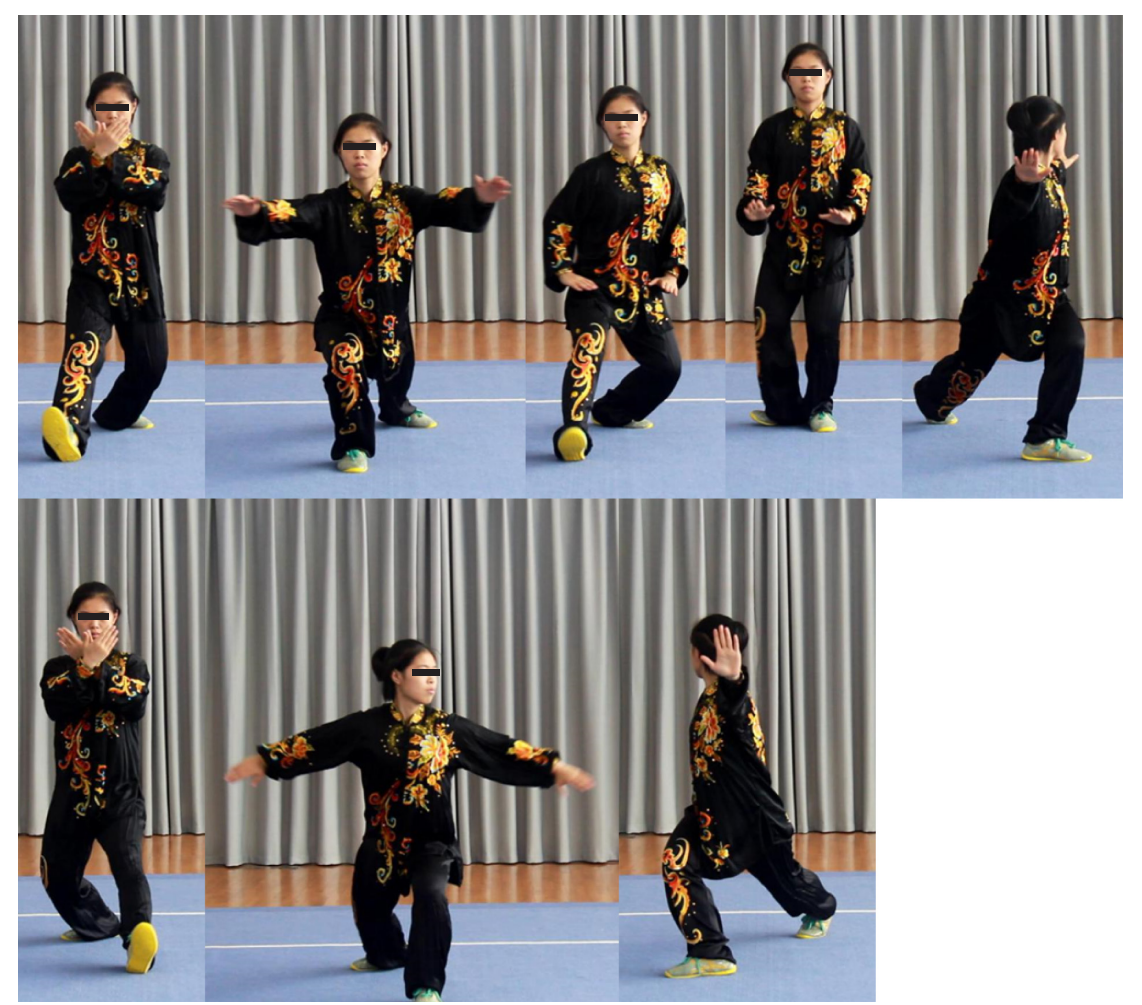

Figure 20 Ba Duan Jin (4 style). The detailed actions of Ba Duan Jin: Look back to treat five strains and seven impairments.
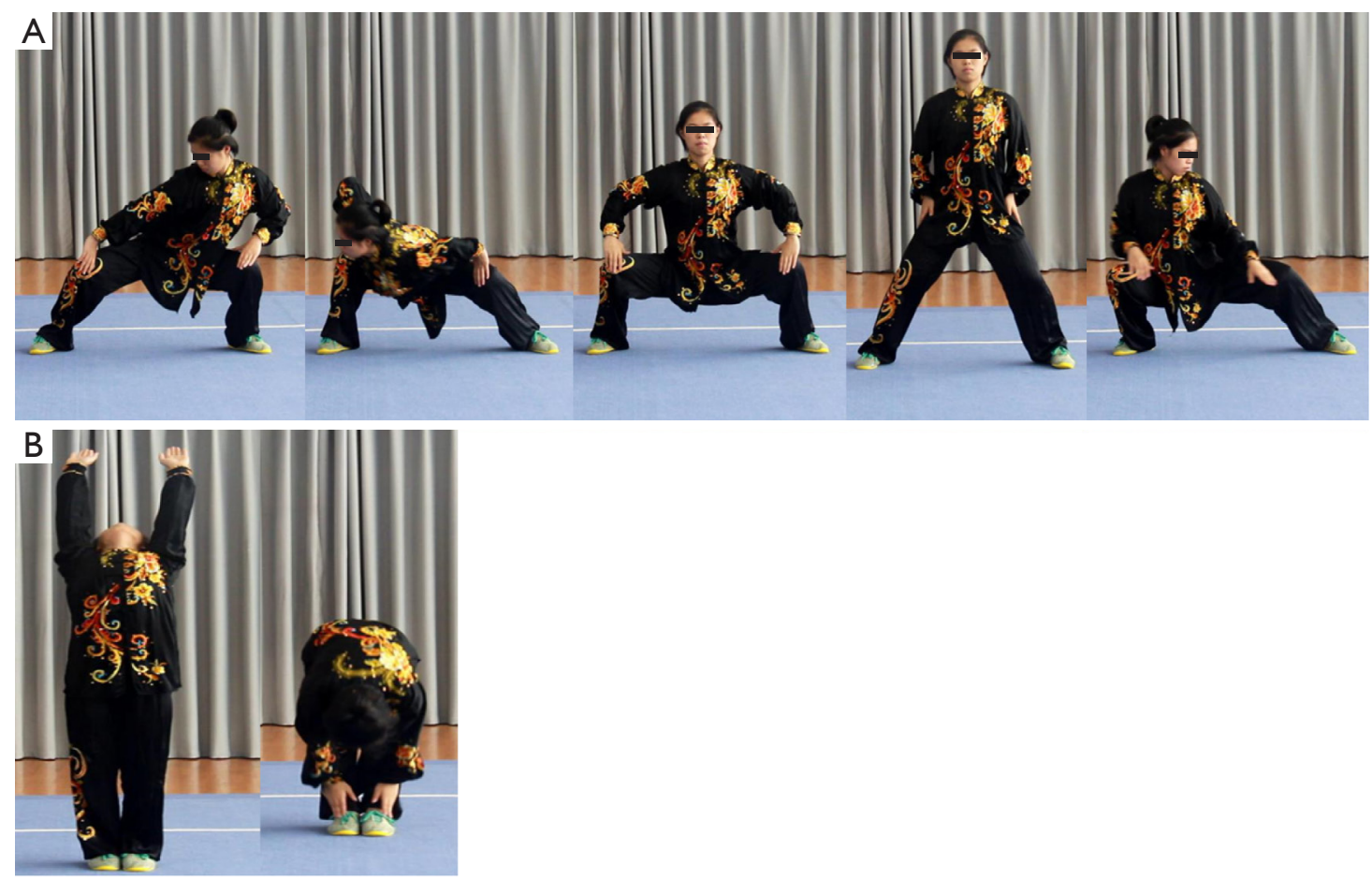

Figure 21 Ba Duan Jin (5-6 styles). The detailed actions of Ba Duan Jin: (A) Swinging the Head and Lowering the Body to Clear Heart Fire. (B) Pull toes with hands to reinfore the kidney (Shen) and waist. 

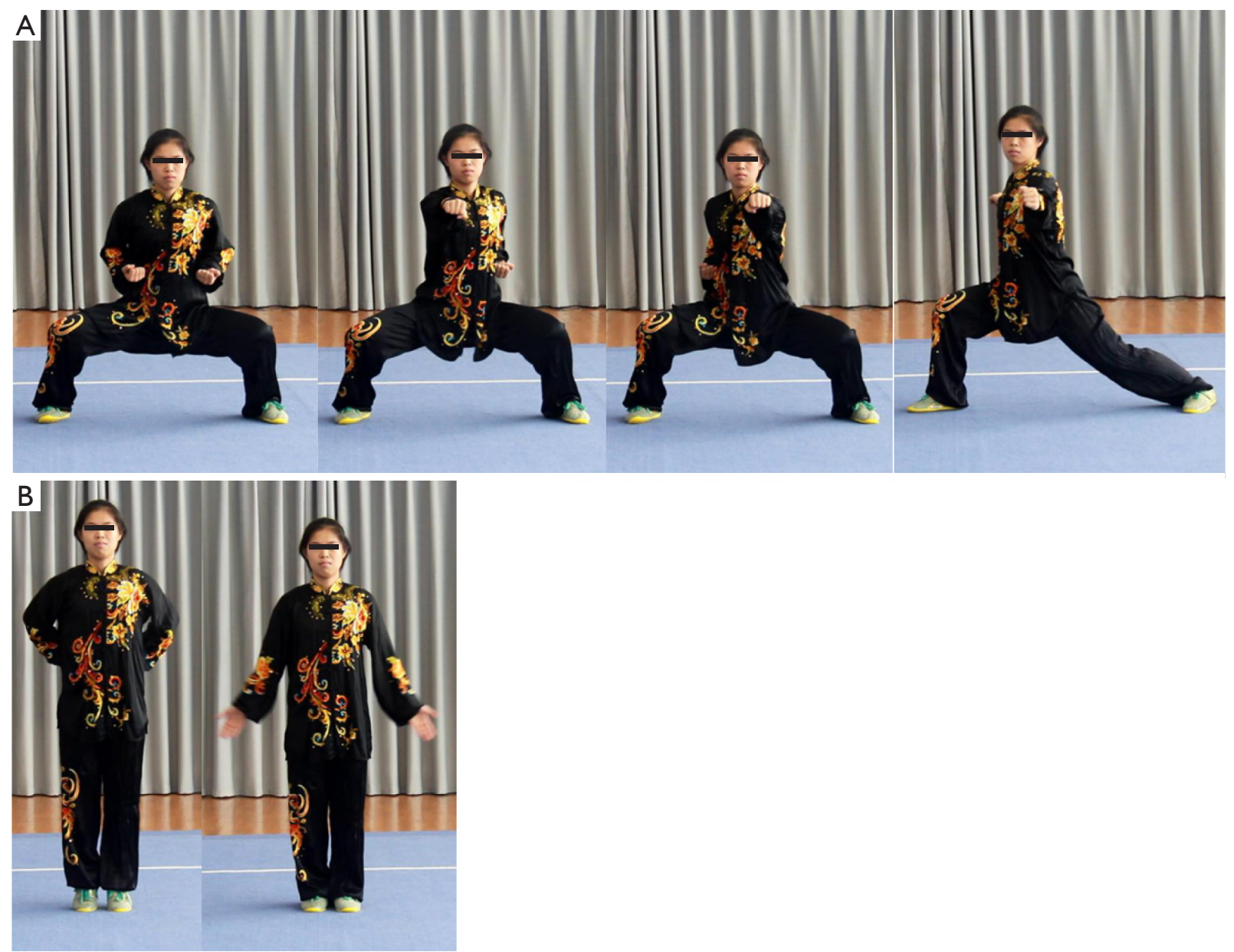

Figure 22 Ba Duan Jin 7-8 styles). The detailed actions of Ba Duan Jin: (A) Clench one's fist and glare to increase strength. (B) Rise and fall on tiptoe seven times to treat all diseases.

the heart, and Si and Chui sound training in the autumn and winter can protect the lungs and kidneys. Practicing $\mathrm{Hu}$ sound training throughout the year improves spleen function and $\mathrm{Xi}$ sound training protects the tri-jiao from heat. The hair must be combed frequently and the breath should be gentle, the teeth should be knocked several times a day, and the saliva should be swallowed. Rubbing the face often with the hands can prolong life. Detailed Liu Zi Jue movements are shown in Figure 23.

\section{Conclusions}

At present, the pulmonary exercise rehabilitation of COPD patients is still in the exploratory stage, and there is still no unified evaluation standard for the curative effect of existing exercise prescriptions. Therefore, further multi-center clinical research is still needed. TCM exercise rehabilitation therapies have a long history. In the long-term practice, the benefits of TCM exercise rehabilitation have proved to be extremely beneficial. And its low dependence on medical equipment can greatly reduce the medical expenses of patients with COPD, and it has good sustainability and operability. COPD is a preventable and treatable chronic disease. Exercise rehabilitation is one of its important auxiliary treatments, which can improve the quality of life of patients both physically and psychologically. The guide focused on the assessment process of the basic condition of COPD patients and the selection of exercise rehabilitation prescriptions in China and the West. It provided a systematic and operational plan for further clinical research on pulmonary exercise rehabilitation intervention for COPD patients. 


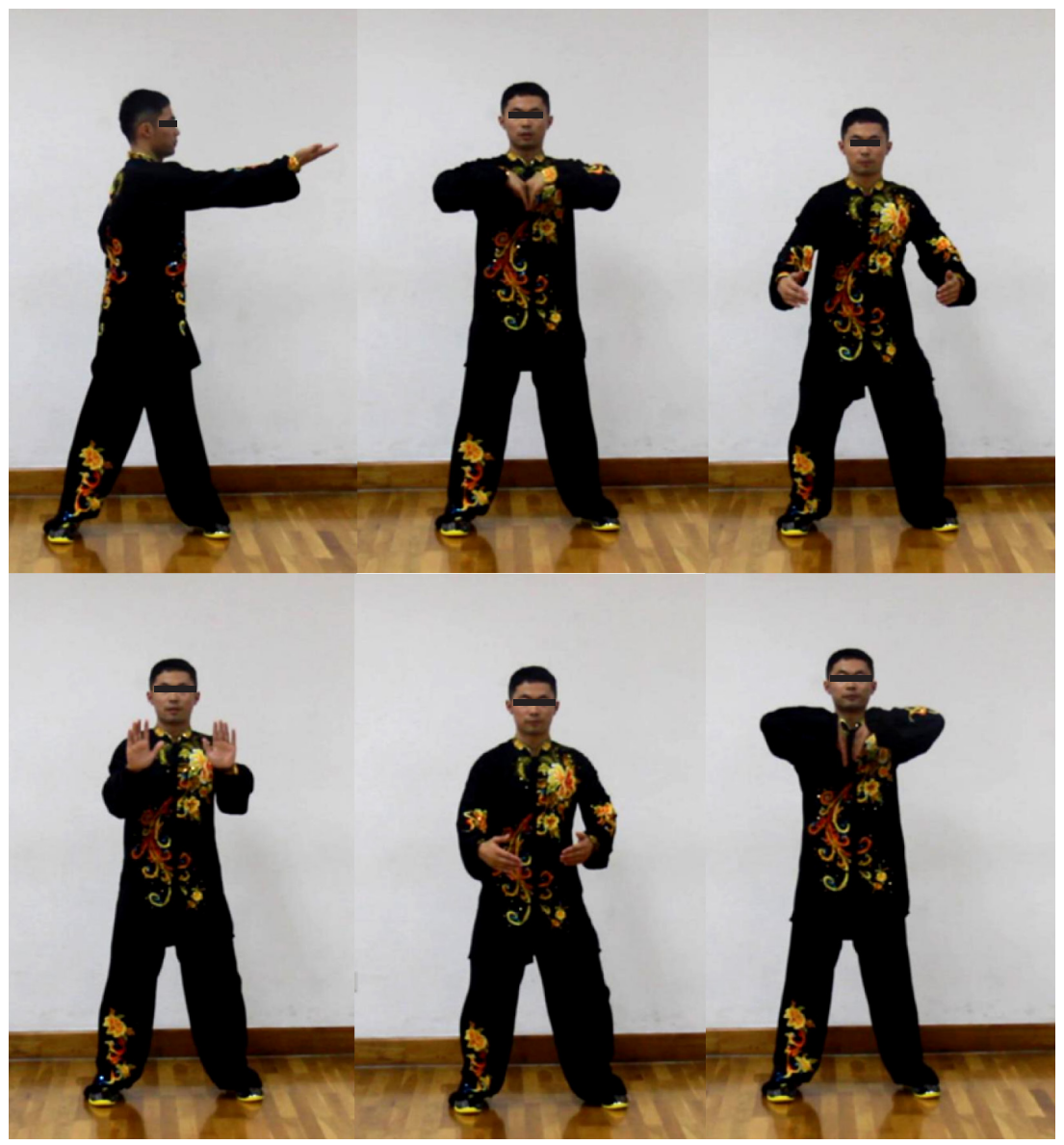

Figure $23 \mathrm{Liu} \mathrm{Zi}$ Jue. From left to right, from top to bottom, the sequence of movements was Xu Sounding, He sounding, Hu sounding, Xi sounding, Chui sounding, Xi sounding.

\section{Acknowledgments}

The guidelines were cooperated with the China COPD Respiratory Rehabilitation Writing Expert Group with integrating Traditional Chinese and Western medicine, the Respiratory Disease Rehabilitation Professional Committee of China Medical Education Association. Members of the China COPD Respiratory Rehabilitation Writing Expert Group with integrating Traditional Chinese and Western medicine, the Respiratory Disease Rehabilitation Professional Committee of China Medical Education Association (listed in alphabetical order), is shown below: Guangxi Li, Department of Pulmonary Medicine, Guang'anmen Hospital, Chinese Academy of Chinese Medical Sciences, Beijing 100053, China; Shanqun Li, Department of Pulmonary medicine, Zhongshan Hospital, Fudan University, Shanghai 200032, China; Xiao Lu, Department of Rehabilitation, the First Affiliated Hospital with Nanjing Medical University, Nanjing 210029, China; Liyan Jiang, Department of Pulmonary Medicine, Shanghai Chest Hospital, Shanghai Jiao Tong University, Shanghai 200030, China; Qing Miao, Department of Pulmonary Medicine, Xiyuan Hospital, Chinese Academy of Traditional Chinese Medical, Beijing 100091, China; Yulian Zhu, Department of rehabilitation, Huashan Hospital, Fudan University, Shanghai 200040, China; Wei Wang, Department of Pulmonary Medicine, the First Affiliated Hospital of China Medical University, Shenyang 110001, China; Kaifeng Xu, Department of Respiratory and Critical Care Medicine, Peking Union Medical College Hospital, Chinese Academy of Chinese Medical Sciences, Beijing 100730, China; Lihua Xing, Department of Respiratory and Critical Care Medicine, the First Affiliated Hospital of Zhengzhou University, Zhengzhou 450052, China; Jin Yan, Department of Nursing, the Third Xiangya Hospital 
of Central South University, Changsha 410013, China; Linfu Zhou, Department of Respiratory and Critical Care Medicine, the First Affiliated Hospital of Nanjing Medical University, Nanjing 210029, China; Wei Zhang, Department of Respiratory and Critical Care Medicine, Shuguang Hospital, Shanghai University of Traditional Chinese Medicine, Shanghai 200120, China; Ziwen Zhao, Department of Pulmonary Medicine, Guangzhou First People's Hospital, Guangzhou 510180, China; Di Gong, Department of Pulmonary Medicine, Zhongshan Hospital, Fudan University, Shanghai 200032, China; Lin Ma, Department of Pulmonary Medicine, the First Affiliated Hospital of Nanchang University, Nanchang 330006, China.

Funding: The National Key Research and Development Program of China (No. 2018YFC1313600), the National Natural Science Foundation of China (No. 81570081, 81770083, 82070094) and the Technical Standard Project of Shanghai Science and Technology Innovation Action Plan (No.20DZ2202300).

\section{Footnote}

Reporting Checklist: The authors have completed the RIGHT reporting checklist. Available at https://dx.doi. org/10.21037/jtd-21-431

Conflicts of Interest: All authors have completed the ICMJE uniform disclosure form (available at https://dx.doi. org/10.21037/jtd-21-431). The authors have no conflicts of interest to declare.

Ethical Statement: The authors are accountable for all aspects of the work in ensuring that questions related to the accuracy or integrity of any part of the work are appropriately investigated and resolved.

Open Access Statement: This is an Open Access article distributed in accordance with the Creative Commons Attribution-NonCommercial-NoDerivs 4.0 International License (CC BY-NC-ND 4.0), which permits the noncommercial replication and distribution of the article with the strict proviso that no changes or edits are made and the original work is properly cited (including links to both the formal publication through the relevant DOI and the license). See: https://creativecommons.org/licenses/by-nc-nd/4.0/.

\section{References}

1. Wang C, Xu J, Yang L, et al. Prevalence and risk factors of chronic obstructive pulmonary disease in China (the China Pulmonary Health CPH study): a national cross-sectional study. Lancet 2018;391:1706-17.

2. Wedzicha JA Ers Co-Chair, Miravitlles M, Hurst JR, et al. Management of COPD exacerbations: a European Respiratory Society/American Thoracic Society guideline. Eur Respir J 2017;49:1600791.

3. Barreiro E, Bustamante V, Cejudo P, et al. Guidelines for the evaluation and treatment of muscle dysfunction in patients with chronic obstructive pulmonary disease. Arch Bronconeumol 2015;51:384-95.

4. Waschki B, Kirsten A, Holz O, et al. Physical activity is the strongest predictor of all-cause mortality in patients with COPD: a prospective cohort study. Chest 2011;140:331-42.

5. Rochester CL, Vogiatzis I, Holland AE, et al. An Official American Thoracic Society/European Respiratory Society Policy Statement: Enhancing Implementation, Use, and Delivery of Pulmonary Rehabilitation. Am J Respir Crit Care Med 2015;192:1373-86.

6. McCarthy B, Casey D, Devane D, et al. Pulmonary rehabilitation for chronic obstructive pulmonary disease. Cochrane Database Syst Rev 2015;CD003793.

7. Maltais F, Decramer M, Casaburi R, et al. An official American Thoracic Society/European Respiratory Society statement: update on limb muscle dysfunction in chronic obstructive pulmonary disease. Am J Respir Crit Care Med 2014;189:e15-62.

8. Spruit MA, Singh SJ, Garvey C, et al. An official American Thoracic Society/European Respiratory Society statement: key concepts and advances in pulmonary rehabilitation. Am J Respir Crit Care Med 2013;188:e13-64.

9. Jones SE, Maddocks M, Kon SS, et al. Sarcopenia in COPD: prevalence, clinical correlates and response to pulmonary rehabilitation. Thorax 2015;70:213-8.

10. Vogelmeier CF, Criner GJ, Martinez FJ, et al. Global Strategy for the Diagnosis, Management and Prevention of Chronic Obstructive Lung Disease 2017 Report: GOLD Executive Summary. Respirology 2017;22:575-601.

11. Qaseem A, Wilt TJ, Weinberger SE, et al. Diagnosis and management of stable chronic obstructive pulmonary disease: a clinical practice guideline update from the American College of Physicians, American College of Chest Physicians, American Thoracic Society, and European Respiratory Society. Ann Intern Med 
2011;155:179-91.

12. Ping Z, LW, Fan Z. Consensus of Chinese experts on exercise rehabilitation for patients with stable coronary heart disease aged 75 years and above. Zhong Hua LaoNian Yi Xu Za Zhi 2017;36:599-607.

13. Vogiatzis I, Terzis G, Stratakos G, et al. Effect of pulmonary rehabilitation on peripheral muscle fiber remodeling in patients with COPD in GOLD stages II to IV. Chest 2011;140:744-52.

14. Puente-Maestu L, Palange P, Casaburi R, et al. Use of exercise testing in the evaluation of interventional efficacy: an official ERS statement. Eur Respir J 2016;47:429-60.

15. Schols AM. Nutrition in chronic obstructive pulmonary disease. Curr Opin Pulm Med 2000;6:110-5.

16. Bolton CE, Bevan-Smith EF, Blakey JD, et al. British Thoracic Society guideline on pulmonary rehabilitation in adults. Thorax 2013;68 Suppl 2:ii1-30.

17. Garber CE, Blissmer B, Deschenes MR, et al. American College of Sports Medicine position stand. Quantity and quality of exercise for developing and maintaining cardiorespiratory, musculoskeletal, and neuromotor fitness in apparently healthy adults: guidance for prescribing exercise. Med Sci Sports Exerc 2011;43:1334-59.

18. Luan X, Tian X, Zhang H, et al. Exercise as a prescription for patients with various diseases. J Sport Health Sci 2019;8:422-41.

19. Garvey C, Bayles MP, Hamm LF, et al. Pulmonary Rehabilitation Exercise Prescription in Chronic Obstructive Pulmonary Disease: Review of Selected Guidelines: AN OFFICIAL STATEMENT FROM THE AMERICAN ASSOCIATION OF CARDIOVASCULAR AND PULMONARY REHABILITATION. J Cardiopulm Rehabil Prev 2016;36:75-83.

20. Machado A, Quadflieg K, Oliveira A, et al. Exercise Training in Patients with Chronic Respiratory Diseases: Are Cardiovascular Comorbidities and Outcomes Taken into Account?-A Systematic Review. J Clin Med 2019;8:1458.

21. Gloeckl R, Marinov B, Pitta F. Practical recommendations for exercise training in patients with COPD. Eur Respir Rev 2013;22:178-86.

22. Zambom-Ferraresi F, Cebollero P, Gorostiaga EM, et al. Effects of Combined Resistance and Endurance Training Versus Resistance Training Alone on Strength, Exercise Capacity, and Quality of Life in Patients With COPD. J Cardiopulm Rehabil Prev 2015;35:446-53.

23. Janaudis-Ferreira T, Hill K, Goldstein RS, et al. Relationship and responsiveness of three upper-limb tests in patients with chronic obstructive pulmonary disease. Physiother Can 2013;65:40-3.

24. Ries AL, Bauldoff GS, Carlin BW, et al. Pulmonary Rehabilitation: Joint ACCP/AACVPR Evidence-Based Clinical Practice Guidelines. Chest 2007;131:4S-42S.

25. McKeough ZJ, Velloso M, Lima VP, et al. Upper limb exercise training for COPD. Cochrane Database Syst Rev 2016;11:CD011434.

26. Kruapanich C, Tantisuwat A, Thaveeratitham P, et al. Effects of Different Modes of Upper Limb Training in Individuals With Chronic Obstructive Pulmonary Disease: A Systematic Review and Meta-Analysis. Ann Rehabil Med 2019;43:592-614.

27. Gigliotti F, Coli C, Bianchi R, et al. Arm exercise and hyperinflation in patients with COPD: effect of arm training. Chest 2005;128:1225-32.

28. Vatwani A. Pursed Lip Breathing Exercise to Reduce Shortness of Breath. Arch Phys Med Rehabil 2019;100:189-90.

29. Mayer AF, Karloh M, Dos Santos K, et al. Effects of acute use of pursed-lips breathing during exercise in patients with COPD: a systematic review and meta-analysis. Physiotherapy 2018;104:9-17.

30. Nield MA, Soo Hoo GW, Roper JM, et al. Efficacy of pursed-lips breathing: a breathing pattern retraining strategy for dyspnea reduction. J Cardiopulm Rehabil Prev 2007;27:237-44.

31. Beaumont M, Forget P, Couturaud F, et al. Effects of inspiratory muscle training in COPD patients: A systematic review and meta-analysis. Clin Respir J 2018;12:2178-88.

32. Güell MR, Cejudo P, Rodríguez-Trigo G, et al. Standards for quality care in respiratory rehabilitation in patients with chronic pulmonary disease. Quality Healthcare Committee. Spanish Society of Pneumology and Thoracic Surgery (SEPAR). Arch Bronconeumol 2012;48:396-404.

33. Zheng W, Li M, Hong Y, et al. Traditional Chinese exercise (TCE) on pulmonary rehabilitation in patients with stable chronic obstructive pulmonary disease: Protocol for a systematic review and network metaanalysis. Medicine (Baltimore) 2019;98:e16299.

34. Luo X, Zhang J, Castelberg R, et al. The Effects of Traditional Chinese Exercise in Patients with Chronic Obstructive Pulmonary Disease: A Meta-Analysis. PLoS One 2016;11:e0161564.

35. Lan C, Chen SY, Lai JS. Relative exercise intensity of Tai Chi Chuan is similar in different ages and gender. Am J Chin Med 2004;32:151-60. 
36. Ngai SP, Jones AY, Tam WW. Tai Chi for chronic obstructive pulmonary disease (COPD). Cochrane Database Syst Rev 2016;CD009953.

37. Liu SJ, Ren Z, Wang L, et al. Mind-Body (Baduanjin) Exercise Prescription for Chronic Obstructive Pulmonary
Disease: A Systematic Review with Meta-Analysis. Int J Environ Res Public Health 2018;15:1830.

38. Xiao CM, Zhuang YC. Efficacy of Liuzijue Qigong in Individuals with Chronic Obstructive Pulmonary Disease in Remission. J Am Geriatr Soc 2015;63:1420-5.

Cite this article as: Chen X, Gong D, Huang H, Wang K, Zhang W, Li S; on behalf of the China COPD Respiratory Rehabilitation Writing Expert Group with integrating Traditional Chinese and Western medicine, the Respiratory Disease Rehabilitation Professional Committee of China Medical Education Association. Expert consensus and operational guidelines on exercise rehabilitation of chronic obstructive pulmonary disease with integrating traditional Chinese medicine and Western medicine. J Thorac Dis 2021;13(6):3323-3346. doi: 10.21037/jtd-21-431 
Supplementary

Table S1 Borg dyspnea scale

\begin{tabular}{lc}
\hline Grade & Degree \\
\hline 0 & None \\
0.5 & Extremely slight (just noticeable) \\
1 & Very slight \\
2 & Slight \\
3 & Moderate \\
4 & Somewhat severe \\
5 & Severe \\
6 & Between 5 and 7 \\
7 & Very severe \\
8 & Between 7 and 9 \\
9 & Extremely severe \\
10 & Maximal \\
\hline
\end{tabular}

Table S2 MMT grading standard

\begin{tabular}{lrr}
\hline Grade & Definition & Percentage of normal strength \\
\hline 0 & Undetectable muscle contraction & 0 \\
1 & Slight constriction, but no joint movement & 10 \\
2 & Muscle moves the joint when gravity is eliminated & 25 \\
3 & Muscle moves the joint fully against gravity but cannot against resistance & 50 \\
4 & Muscle moves the joint against gravity and moderate resistance & 75 \\
\hline
\end{tabular}

